\title{
CHECK LIST
}

CANADIAN PLANTS.

\section{JAMES M. ViACOON,}

Assistant Boiun " to the Ge logical and Natural History Survey of Cana $x$.

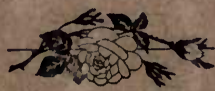

OTTA

PRINTE) BY CUNNINGHAM \& LINDSAY.

1889. 

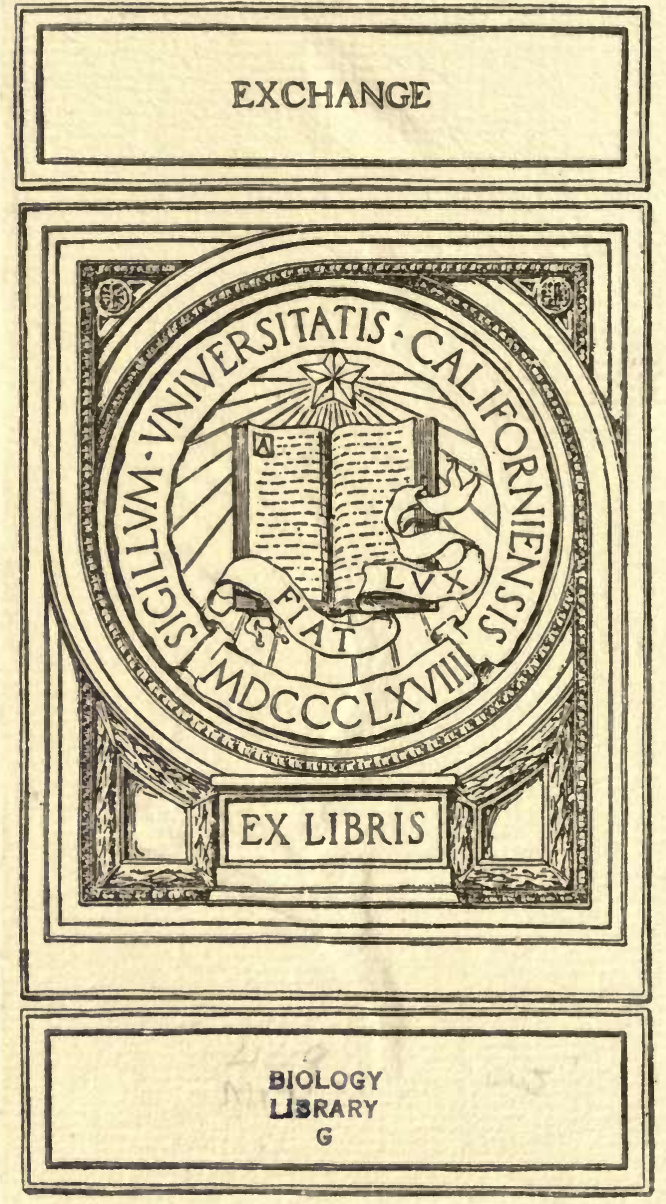


$$
197
$$





\section{CHECK LIST}

OF

\section{CANADIAN PLANTS.}

\section{JAMES M. MACOUN,}

Assistant Botanist to the Geological and Natural History Survey of Canada.

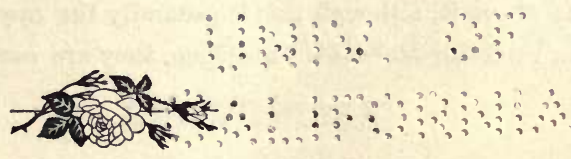

\section{OTTAWA:}

PRINTED BY CUNNINGHAM \& LINDSAY.

1889. 
QK201

M3

BIOLOGY

LBRARY

G

\section{E R R A T A.}

Page 7.-For "Nuphar pygmæa, Salisb." read “Nymphæa pygmæa, Salisb."

" 7.-For "Saraceniaceæ and Saracenia " read "Sarraceniaceæ and Sarracenia."

" 7.-For "Papaver Rheas" read "Papaver Rhæas."

" 16. -For "Gleditschi" read "Gleditschia."

" 18. - For "Sarothammus" read "Sarothamnus."

" 19.-After "Cratægus coccinea, L." insert "var. macrantha."

" 19.-For "Pirus Acuparia" read "Pirus Aucuparia."

" 20.-For "Rosa acicularis, var. Bourgeaniana," read "Bourgeauiana."

" 22.-For "Saxifraga azoides" read "Saxifraga aizoides."

" 23. -For " Epilobium minutum, var. folisum," read "var. foliosum."

" 24.-For "Anthruscus" read "Anthriscus."

" 25.-For "Peucednaum" read "Peucedanum."

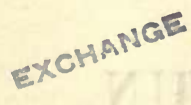

\section{NOTE.}

The basis of this Check-list has been Professor John Macoun's Catalogue of Canadian Plants. Where a name is printed in brackets it signifies, not that it is a synonym of the one immediately above.it, although this is zenerally the case, but that, where specimens have been so named in Professer Macón's cartag the they are now known by the name given in the list.

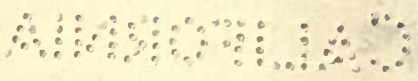




\section{LIST OF CANADIAN PLANTS.}

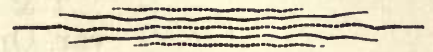

\section{RANUNCULACEA.}

\section{ACONITUIM}

Columbianum, Nutt.

(A. Fischeri, Reich.)

Napellus, L., var. delphinifolium, Seringe.

\section{ACT平A}

alba, Bigel.

spicata, L., var. arguta, Torr. var. rubra, Ait.

\section{ANEMONE}

acutiloba, Lawson.

cylindrica, Gray.

deltoidea, Hook.

dichotoma, L.

Drummondii, Watson.

Hepatica, L.

multifida, Poir.

narcissiflora, $\mathrm{L}$.

nemorosa, $\mathrm{L}$.

nudicaulis, Gray.

parviflora, Michx.

patens, L., var. Nuttalliana, Gray occidentalis, Watson.

Richardsoni, Hook.

Virginiana, L.

\section{ANEIMONELLA}

thalictroides, Spach.

(Thalictrum anemonoides, Michx.)

\section{AQUILEGIA}

brevistyla, Hook.

Canadensis, $\mathrm{L}$.

flavescens, Watson.

formosa, Fisch.

vulgaris, $L$.

\section{CALTHA}

biflora, DC.

(C. palustris, L., var. minima, Regel.)

leptosepala, DC.

natans, Pall.

palustris, L.

var. Sibirica, Regel.

\section{CIMICIFUGA}

elata, Nutt.

racemosa, Nutt.

\section{CLEMATIS}

Douglasii, Hook. ligusticifolia, Nutt.

var. brevifolia, Nutt.

verticillaris, $\mathrm{DC}$.

var. Columbiana, Gray.

Virginiania, L. 
COPTIS

asplenifolia, Salisb.

trifolia, Salisb.

\section{DELPHINIUM}

azureum, Michx.

Consolida, $L$.

Menziesii, DC.

scopulorum, Gray.

variegatum, T. \& $\mathrm{G}$,

\section{HYDRASTIS}

Canadensis, L.

\section{MYOSURUS}

apetalus, Gay. var. lepturus, Gray.

aristatus, $\mathrm{L}$.

minimus, $\mathrm{L}$.

\section{P FONIA}

Brownii, Dougl.

\section{RANUNCULUS}

abortivus, $\mathrm{L}$.

(var. micranthus, Gray.)

acris, $\mathrm{L}$.

affinis, $\mathrm{R} . \mathrm{Br}$.

var. leiocarpus, Trautv.

var. validus, Gray.

(var. cardiophyllus, Gray.)

alismæfolius, Geyer.

ambigens, Watson.

aquatilis, $\mathrm{L}$. var. heterophyllus, Wat.

(R. hederaceus, L.)

var. trichophyllus, Gray

(var. stagnatilis, DC.)

(var. caespitosus, DC.)

(var. confervoides, Wat.)

bulbosus, $L$.,

Californicus, Benth.

Cymbalaria, Pursh.

(var. alpinus, Hook.)

Eschscholtzii, Schlecht.
(R. nivalis, L., var. Eschscholtzii, Wat.)

fascicularis, Muhl.

Flammula, L., var. intermedius, Hook.

var. reptans, Meyer.

glaberrimus, Hook.

glacialis, L.

hispidus, Mx., DC., Hook.

(R. repens, L., var. hispidus, T \& G.)

var. Oreganus, Gray.

Hookeri, Regel.

hyperboreus, Rottb.

Lapponicus, $\mathrm{L}$.

multifidus, Pursh.

(var. B. Wat.)

(var. repens, Wat.)

var. terrestris, Gray.

muricatus, $L$.

nivalis, $\mathrm{L}$.

occidentalis, Nutt.

(R. Nelsoni, Gray.)

var, robustus, Gray.

var. tenellus, Gray.

(R. Nelsoni, Gray, var. tenellus, Gray.)

orthorhynchus, Hook., var. stenophyllus, Gray.

Pallasii, Schlecht.

Pennsylvanicus, L. f.

pygmæus, Wahl.

recurvatus, Poir.

repens, $\mathrm{L}$.

(var. nitidus, Chapm.)

rhomboideus Raf.

sceleratus, L.

\section{THALICTRUIM}

alpinum, $\mathrm{L}$.

dioicum, $\mathrm{L}$.

occidentale, Gray.

polygamum, Munl.

(T. Cornuti, L.)

purpurascens, L.

(var. ceriferum, Austin.) 


\section{TRUTVETTERIA}

grandis, Nutt.

\section{TROLLIUS}

laxus, Salisb.

\section{MAGNOLIACEAE.}

\section{LIRIODENDRON}

Tulipifera, L.

MAGNOLIA

acuminata, L.

\section{ANONACEA.}

\section{ASIMINA}

triloba, Dunal.

\section{MENISPERMACEA.}

MENISPERIMUM

Canadense, $\mathrm{L}$.

\section{BERBERIDACEA.}

\section{ACHLYS}

triphylla, DC.

\section{BERBERIS}

Aquifolium, Pursh.

nervosa, Pursh.

repens, Lindl.

vulgaris, $L$.

\section{CAULOPHYLLUM}

thalictroides, Michx.

\section{JEFFERSONIA}

diphylla, Pers.
PODOPHYLLUM

peltatum, L.

\section{VANCOUVERIA}

hexandra, Morr. \& Decne.

NYMPHAACEA.

BRASENIA

peltata, Pursh.

NELUMBIUIM

luteum, Willd.

NUPHAR

advena, Ait.

Kalmianum, Ait.

(N. pumilum, Smith.)

polysepalum, Engelm.

pygmæa, Salisb. rubrodiscum, Morong,

(N. luteum, Smith.)

\section{NYMPHA A}

odorata, Ait.

var. minor, Sims.

tuberosa, Paine.

SARACENIACEA.

\section{SARACENIA}

purpurea, L.

var. heterophylla, Torr.

\section{PAPAVERACEA.}

\section{CHELIDONIUIM}

majus, $L$.

\section{PAPAVER}

nudicaule, L.

Rheas, $L$.

somniferum, $L$., 


\section{PLATYSTIGMA}

Oreganum, Benth. \& Hook.

\section{SANGUINARIA}

Canadensis, $\mathrm{L}$.

\section{FUMARIACEA.}

\section{ADLUMIA}

cirrhosa, Raf.

\section{CORYDALIS}

aurea, Willd.

var. occidentalis, Gray. flavula, DC.

\section{DICENTRA}

Canadensis, DC.

Cucullaria, DC.

formosa, DC.

\section{FUMARIA}

officinalis, $L$.

parviflora, $L$.

\section{CRUCIFERA.}

\section{ALYSSUM}

calycinum, $L$.

\section{ARABIS}

alpina, L.

Canadensis, $\mathrm{L}$.

canescens, Nutt., var. latifolia, Watson, confinis Wat.

(A. Drummondii, Gray, var.) brachycarpa, Gray.

Drummondii, Gray. hirsuta, Scop.

Holbœellii, Hornem. humifusa, Wat., var. pubescens, Wat.

(A. petræa, Lam.)

lævigata, Poir.

Lyallii, Watson.

lyrata, $\mathrm{L}$.

var. occidentalis, Wat.

(A. petræa, Lam. var. ambigua, Regel,)

perfoliata, Lam.

stricta, Huds.

\section{BARBAREA}

vulgaris, $R$. Br.

var, arcuata, Koch.

var, stricta, Regel.

glauca, Pursh.

pauciflora, Pursh.

Scouleri, Hook.

\section{BRASSICA}

alba, Gray.

campestris, $L$.

nigra, Koch,

Rapa, $L$.

Sinapistrum, Boiss.

\section{BRAYA}

alpina. Sternb., var. Americana, Hook.

var. glabella, Watson. Eschscholtziana, B. \& H. pilosa, Hook.

\section{CAKILE}

Americana, Nutt.

\section{CAMELINA}

sativa, Crantz.

\section{CAPSELLA}

Bursa-pastoris, Maench. divaricata, Walp. 


\section{CARDAIMINE}

angulata, Hook.

bellidifolia, $\mathrm{L}$.

hirsuta, L.

var. sylvatica, Gray.

oligosperma, Nutt.

pratensis, L.

var. occidentalis, Wat. purpurea, Cham. \& Schl. rhomboidea, DC.

var. purpurea, Torr. rotundifolia, Michx.

\section{CHEIRANTHUS}

asper, Cham. \& Schlecht.

pygmæus, Adams.

\section{COCHLEARIA}

Anglica, L.

Danica, L.

offlcinalis, $\mathrm{L}$.

tridactylites, Banks.

\section{DENTARIA}

diphylla, Michx.

heterophylla, Nutt.

laciniata, Muhl.

maxima, Nutt.

tenella, Pursh.

\section{DIPLOTAXIS}

muralis $D C$.

tenuifolia, $D C$.

\section{DRABA}

alpina, L.

var. algida, Regel.

var. (?) corymbosa, Durand.

var. glacialis, Dickie.

var. hebecarpa, Lindb.

var. (?) mircropetala, Durand, androsacea, Wahl. arabisans, Michx. aurea, Vahl.
Caroliniana, Walt. crassifolia, Grah. hirta, L.

hyperborea, Desv. var. siliquosa, Gray. incana, $\mathrm{L}$.

var. borealis, T. \& G.

var. confusa, Poir.

nemorosa, L, var. hebecarpa, Lindb.

var. leiocarpa, Lindb. rupestris, $\mathrm{R}$. Br. stellata, Jacq.

var. hebecarpa, DC.

var. Johannis, Regel.

var. nivalis, Regel. stenoloba, Ledeb.

verna, $\mathrm{L}$.

\section{EUTREMA}

Edwardsii, R. Br.

\section{ERYSIMUIM}

asperum, DC. cheiranthoides, L. parviflorum, Nutt. orientale, $R$. Br.

\section{HESPERIS}

matronalis, $L$.

\section{LEPIDIUIM}

campestre, $R . B r$.

Draba, $L$.

intermedium, Gray.

Menziesii, DC.

ruderale, $L$.

sativum, $L$.

Virginicum, L.

\section{NASTURTIUIM}

amphibium, R. Br. Armoracia, Fries. curvisiliqua, Nutt. 
lacustre, Gray.

obtusum, Nutt.

officinale, $R$. Br.

palustre, DC.

var, hispidum, F. \& M. trachycarpum, Gray.

\section{NESLIA}

paniculata, Desv.

\section{PARRYA}

arctica, $\mathrm{R}$. Br. arenicola, Hook. f. nudicaulis, var. aspera, Regel.

var. glabra, Regel.

\section{PHYSARIA}

didymocarpa, Gray.

\section{PLATYSPERIMUMI}

scapigerum, Hook.

\section{RAPHANUS}

Raphanistrum, $L$. sativus, $L$.

\section{SENEBIERA}

Coronopus, Poir. didyma, Pers.

\section{SISYIMBRIUIM}

acutangulum, $D C$. canescens, Nutt.

var. brachycarpum, Wats.

humile, C. A. Meyer.

incisum. Engelm.

var. filipes, Gray.

var, Hartwegianum, Wats.

linifolium, Nutt.

officinale, Scop.

salsugineum, Pall.

Sophia, $L$.

var. (S.sophioides, Fisch.)

Thaliana, Gay.
SMELOWSKIA

calycina, C. A. Meyer.

SUBULARIA

aquatica, L.

\section{THELYPODIUM}

pinnatifidum, Watson.

\section{THLASPI}

alpestre, L.

arvense, $L$.

\section{THYSANOCARPUS}

curvipes, Hook.

pusillus, Hook.

\section{VESICARIA}

alpina, Nutt.

arctica, Richards.

Ludoviciana, DC.

\section{CAPPARIDACEA.}

\section{CLEOME}

integrifolia, T. \& G.

lutea, Hook.

\section{POLANISIA}

graveolens, Raf.

uniglandulosa, DC.

CISTACEA.

HELIANTHEMUM

Canadense, Michx.

\section{HUDSONIA}

ericoides, L.

tomentosa, Nutt. 


\section{LECHEA}

major, Michx.

minor, Walt.

thymifolia, Pursh.

\section{VIOLACEA.}

\section{SOLEA}

concolor, Ging.

(Ionidium concolor, Benth. \& Hook.

\section{VIOLA}

biflora, L.

blanda, Willd.

var. renifolia, Gray.

(V. renifolia. Gray.)

Canadensis, $\mathrm{L}$.

canina, L., var. adunca, Gray.

(var. longipes, Gray.)

(var. rupestris, Regel.)

var. Muhlenbergii, Gray.

(var. sylvestris, Regel.)

glabella, Nutt.

lanceolata, $\mathrm{L}$.

Langsdorffii, Fisch.

Nuttallii, Pursh.

(var. linguæfolia, Nutt.)

odorata, $L$.

palmata, L.

(V. cucullata, Ait, var. palmata, Gray.)

var. cucullata, Gray.

(V. cucullata, Ait.)

(V. cucullata, Ait, var. cordata, Gray.)

palustris, L.

pedata, L.

pedatifida, Don.

(V. delphinifolia, Nutt.)

præmorsa, Dougl.

(V. Nuttallii, Pursh, var. præmorsa Wat.)

primulifolia, L. pubescens, Ait. (var. eriocarpa, Nutt.)

var. scabriuscula, T. \& G.

rostrata, Muhl.

rotundifolia, Michx.

sagittata, Ait.

sarmentosa, Dougl.

Selkirkii, Pursh.

striata, Ait.

tricolor, L. var. arvensis, DC.

\section{POLYGALACEA.}

POLYGALA

incarnata, $\mathrm{L}$.

paucifolia, Willd.

polygama, Walt.

sanguinea, $\mathrm{L}$.

Senega, L.

verticillata, $\mathrm{L}$.

\section{CARYOPHYLLACEA.}

\section{ARENARIA}

arctica, Stev.

var. breviscapa, Regel.

biflora, Watson, var. obtusa, Wat. capillaris, Poir,, var. nardifolia, Regel.

congesta, Nutt., var. subcongesta,

Watson.

Grœnlandica, Spreng.

lateriflora, $\mathrm{L}$.

macrocarpa, Pursh.

macrophylla, Hook.

Michauxii, Hook. f.

peploides, L.

var. oblongifolia. Watson. physodes, DC.

pungens, Nutt. serpyllifolia, $L$. stricta, Watson. tenella, Nutt. verna, $\mathrm{L}$.

var. hirta, Watson.

var. rubella, Hook. f. 


\section{CERASTIUIM}

alpinum, L.

var. Behringianum, Regel.

var. Fischerianum, T. \& G.

var. glabratum, Hook.

var. latifolium, Smith. (?)

arvense, $\mathrm{L}$.

nutans, Raf.

oblongifolium, Torr.

pilosum, Ledeb.

trigynum, Vill.

viscosum, $L$.

vulgatum, L.

\section{DIANTHUS}

alpinus, L., var. repens, Regel. Armeria, $L$.

\section{LEPIGONUM (Spergularia.)}

macrothecum, Fisch \& Mey. medium, Fries.

var. macrocarpum, Watson. rubrum, Fries.

var. campestris, Gray. salinum, Fries.

\section{LYCHNIS}

affinis, Vahl.

alpina, L.

apetala, $\mathrm{L}$.

var. glabra, Regel.

dioica, $L$.

Drummondii, Watson.

elata, Watson. (?)

Githago, Lam.

F'los-cuculi, $L$.

montana, Watson.

vespertina, Sibth.

\section{SAGINA}

decumbens, T. \& G.

Linnæi, Presl.

nodosa, E. Meyer. occidentalis, Watson.

procumbens, L.

\section{SAPONARIA}

officinalis, $L$.

Vaccaria, $L$.

\section{SILENE}

acaulis, $\mathrm{L}$.

antirrhina, L.

Armeria, $L$.

Douglasii, Hook.

Gallica, L. inflata, Sm.

Menziesii, Hook. multicaulis, Nutt. noctiflora, $L$. nocturna, $L$.

Pennsylvanica, Michx. (?)

Scouleri, Hook.

stellata, Ait. f.

Virginica, L.

\section{SPERGULA}

arvensis, $L$.

\section{STELLARIA}

borealis, Bigel.

var. alpestris, Gray.

calycantha, Bongard. (?)

crassifolia, Ehrh.

crispa, Cham. \& Schlecht.

graminea, L.

gracilis, Richards.

humifusa, Rottb.

longifolia, Muhl.

longipes, Goldie.

var. Edwardsii, T \& G.

var. læta, T. \& G.

var. minor, Hook.

media, Sm.

nitens, Nutt.

obtusa, Eng.

uliginosa, Murr.

umbellata, Turez. 


\section{PARONYCHIEAE.}

\section{ANYCHIA}

dichotoma, Michx.

PARONYCHIA

sessiliflora, Nutt.

SCLERANTHUS

annuus, $L$.

\section{PORTULACACEA.}

\section{CALANDRINIA}

Menziesii, Hook.

pygmæa, Gray.

Vancouverensis, Macoun,

\section{CLAYTONIA}

Caroliniana, Michx. var. sessilifolia, Torr.

Chamissonis, Esch.

dichotoma, Nutt.

exigua, T. \& G.

linearis, Dougl.

megarrhiza, Parry.

parvifolia, Moc.

perfoliata, Donn.

var. parviflora, Torr.

var. spathulata, Watson.

sarmentosa, C. A. Meyer.

Sibirica, L.

Virginica, L.

\section{LEWISIA}

rediviva, Pursh.

\section{MONTIA}

fontana, L.

\section{PORTULACA}

oleracea, $L$.
SPRAGUEA

umbellata, Torr.

\section{TALINUIM}

spinescens, Torr.

\section{ELATINEA.}

\section{ELATINE}

Americana, Arnott.

\section{HYPERICACEA.}

\section{ELODEA}

campanulata, Pursh.

(E. Virginica, Nutt.)

\section{HYPERICUIM}

anagalloides, Cham. \& Schl.

Ascyron, L.

(H. pyramidatum, Ait.)

Canadense, L.

var. major, Gray.

ellipticum, Hook.

formosum, HBK., var. Scouleri, Coulter.

(H. Scouleri, Hook.)

Kalmianum, L. maculatum, Walt.

(H. corymbosum, Muhl.)

mutilum, L.

nudicaule, Walt.

(H. Sarotha, Michx. perforatum, $L$.

\section{MALVACEA.}

\section{ABUTILON}

Avicenno, Gartn.

\section{HIBISCUS}

Moscheutos, L.

Trionum, $L$. 
MALVA

borealis, Wallm.

crispa, $L$.

moschata, $L$.

rotundifolia, $L$.

sylvestris, $L$.

MALVASTRUIM

coccineum, Gray.

Munroanum, Gray.

\section{SIDA}

hederacea, Torr. (?)

\section{SIDALCEA}

malvæflora, Gray.

Oregana, Gray,

\section{SPH开RALCEA}

acerifolia, Nutt.

(S. rivularis, Torr.)

\section{TILIACEA.}

\section{TIIIA}

Americana, L.

var. pubescens, Loud Europaca, L.

\section{LINACEA.}

\section{LINUMI}

Catharticum, $L$.

perenne, $\mathrm{L}$.

rigidum, Pursh.

striatum, Walt.

sulcatum, Riddell.

usitatissimum, $L$.

Virginianum, L.

\section{GERANIACEA.}

\section{ERODIUM}

cicutarium, L'Her.

moschatum, Willd.

\section{FLFRKEA}

proserpinacoides, Willd.

\section{GERANIUIM}

Carolinianum, L.

dissectum, $L$.

erianthum, DC.

incisum, Nutt.

(G. Fremonti, Torrey.)

maculatum, $\mathrm{L}$.

molle, $L$.

pratense, $L$. pusillum, $L$.

Richardsoni, Fisch. \& Mey. Robertianum, L.

\section{IMPATIENS}

fulva, Nutt.

pallida, Nutt.

\section{LIMNANTHES}

Douglasii, R. Br.

\section{OXALIS}

Acetosella, L. corniculata, L. var. stricta, Sav. Oregana, Nutt.

\section{RUTACEA.}

\section{PTELEA}

trifoliata, L.

\section{XANTHOXYLUIM}

Americanum, Mill. 


\section{ILICINEA.}

\section{ILEX}

glabra, Gray.

opaca, Ait. (?)

verticillata, Gray.

\section{NEMOPANTHES}

Canadensis, DC.

\section{CELASTRACEA.}

\section{CELASTRUS}

scandens, L.

\section{EUONYMIUS}

Americanus, L., var. obovatus,

T. \& G.

atropurpumus, Jacq.

\section{PACHYSTIMA}

Myrsinites, Raf.

\section{RHAMNACEA.}

\section{CEANOTHUS}

Americanus, $\mathrm{L}$. ovatus, Desf. sanguineus, Pursh. velutinus, Dougl.

var. lævigatus, T. \& G.

\section{RHAMNUS}

alnifolia, L'Her.

Cathartica, $L$.

Purshiana, DC

\section{VITACEA.}

\section{AMPELOPSIS}

quinquefolia, Michx.

\section{VITIS}

æestivalis, Michx.

(V. Labrusca, L.) cordifolia, Lam. riparia, Michx.

\section{SAPINDACEA.}

\section{ACER}

circinatum, Pursh, dasycarpum, Ehrh.

glabrum, Torr.

macrophyllum, Pursh.

Pennsylvanicum, $\mathrm{L}$.

rubrum, L.

saccharinum, Wangenh. var. nigrum, T. \& G. spicatum, Lam.

\section{ÆSCULUS}

Hippocastanum, $L$.

\section{NEGUNDO}

aceroides, Mœench.

\section{STAPHYLEA}

trifolia, L.

\section{ANACARDIACEAE.}

\section{RHUS}

aromatica, Ait.

var. trilobata, Gray.

copallina, L.

diversiloba, T. \& G.

glabra, L.

Toxicodendron, $\mathrm{L}$.

var. radicans, Torr.

typhina, L.

venenata, $\mathrm{DC}$. 


\section{LEGUMINOSA.}

\section{AMORPHA}

canescens, Nutt.

fruticosa, L.

microphylla, Pursh.

\section{AMPHICARP AFA}

monoica, Dll.

\section{APIOS}

tuberosa, Mœench.

\section{ASTRAGALUS}

aboriginum, Richard.

adsurgens, Pall.

alpinus, L.

Beckwithii, T. \& G.

bisulcatus, Gray.

Bourgovii, Gray.

cæspitosus, Gray.

campestris, Gray.

Canadensis, L.

caryocarpus, Ker.

collinus, Dougl,

Cooperi, Gray.

decumbens, Gray.

Drummondii, Dougl.

flexuosus, Dougl.

frigidus, Gray, var: Americanus,

Watson.

var. littoralis, Watson.

Gibbsii, Kellog.

glabriusculus, Gray.

Hallii, Gray.

(A. vaginatus, Rich.)

hypoglottis, L.

Kentrophyta, Gray.

lentiginosus, Dougl.

lotiflorus, Hook.

Lyallii, Gray.

microcystis, Gray.

Missouriensis, Nutt. multiflorus, Gray. oroboides, Hornem, var. Americanus, Gray.

Palliseri, Gray. pauciflorus, Hook. pectinatus, Dougl. polaris, Benth. Purshii, Dougl. racemosus, Pursh. speirocarpus, Gray. triphyllus, Pursh.

\section{BAPTISIA}

leucantha, T. \& G. tinctoria, R. Br.

\section{DESIMODIUIM}

acuminatum, DC.

Canadense, DC.

canescens, DC.

ciliare, DC.

cuspidatum, Hook.

Dillenii, Darlington.

nudiflorum, DC.

paniculatum, DC. pauciflorum, DC. rotundifolium, DC. sessilifolium, T. \& G.

\section{GLEDITSCHIA}

triacanthos, $L$.

\section{GLYCYRRHIZA}

lepidota, Pursh. var. glutinosa, Watson.

\section{GYMNOCLADUS}

Canadensis, Lam.

\section{HEDYSARUM}

boreale, Nutt. var. albiflorum, Macoun. Mackenzii, Richard. 


\section{HOSACIXIA}

bicolor, Dougl.

decumbens, Benth.

parvifiora, Benth.

Purshiana, Benth,

subpinnata, T. \& (

\section{LATHYRUS}

Aphaca, $L$.

maritimus, Bigel.

ochroleucus, Hook.

Oreganus, Watson.

paluster, L.

var. myrtifolius, Gray.

pratensis, $L$.

venosus, Muhl.

\section{LESPEDEZA}

capitata, Michx.

hirta, Ell.

repens, Barton.

reticulata, Pers.

\section{LOTUS}

corniculatus, Koch.

\section{LUPINUS}

arcticus, Watson.

argentus, Pursh, var. argophyllus,

Watson.

aridus, Dougl. (?)

densiflorus, Benth.

Kingii, Watson.

laxiflorus, Dougl.

lepidus, Dougl.

leucophyllus, Dougl.

littoralis, Dougl.

Lyallii, Gray.

micranthus, Dougl, var. bicolor,

Warson.

minimus, Dougl.

Nootkatensis, Doun.

var. Unalaskensis, Watson. ornatus, Dougl. perennis, $\mathrm{L}$.

polyphyllus, Lindl.

pusillus, Pursh. (?)

Sabinii, Dougl. (?)

sericeus, Pursh.

sulphureus, Dougl. (?)

\section{MEDICAGO}

denticulata, Willd.

lupulina, $L$.

maculata, Willd.

sativa, $L$.

\section{MELILOTUS}

alba, Lam.

officinalis, Will,.

parviflora, Desf.

\section{ONOBYCHIS}

sativa, Lam.

\section{ORNITHOPUS}

scorpoides, $D C$.

\section{OXYTROPIS}

arctica, R. Br.

(O. Uralensis, I)C., var. pumila, Ledeb.)

campestris, L., var. cærulea, Koch, (O. campestris, DC.) deflexa, DC.

Lamberti, Pursh.

leucantha, Pers.

Mertensiana, Turez.

monticola, Gray.

nana, Nutt

nigrescens, Fisch.

var. arctobia, Gray.

podocarpa, Gray.

splendens, Dougl.

var. Richardsoni, Hook. viscida, Nutt.

(O. campestris, DC., var. viscida, Watson. 


\section{PETALOSTEMON}

candidus, Michx. violaceus, Michx.

var. pubescens, Gray.

\section{PHASEOLUS}

diversifolius, Pers.

\section{PSORALEA}

argophylla, Pursh. esculenta, Pursh. lanceolata, Pursh. physodes, Dougl.

\section{ROBINIA}

Pseudacacia, $L$. viscosa Vent.

\section{SAROTHAMMUS}

scoparius, Koch

\section{TEPHROSIA}

Virginiana, Pers.

\section{THERIMOPSIS}

montana, Nutt. rhombifolia, Richard.

\section{TRIFOLIUM}

agrarium, $L$.

arvense, $L$.

cyathiferum, Lindl. depauperatum, Desv. fucatum, Lindl. gracilentum, T. \& G. hybridum, L. involucratum, Willd.

var. heterodon, Watson.

longipes, Nutt. medium, $L$. megacephalum, Nutt. microcephalum, Pursh. microdon, Hook. \& Arn. ornithopodioides, $L$. pauciflorum, Nutt.

pratense, $L$.

procumbens, $L$. var. minus, Koch.

reflexum, $\mathrm{L}$.

repens, $L$.

resupinatum, $L$.

tridentatum, Lindl.

\section{TRIGONELLA}

corniculata, $L$.

\section{ULEX}

Europoeus, $L$.

\section{VICIA}

Americana, Muhl. var. linearis, Watson. var. truncata, Brewer. Caroliniana, Walter. Cracca, L. hirsuta, Koch. sativa, $L$. var. angustifolia, Ser. tetrasperma, Loisel.

\section{ROSACEA.}

\section{AGRIMONIA}

Eupatoria, L. var. parviflora, Hook. parviflora, Ait.

\section{ALCHEMILLA}

arvensis, Scop. vulgaris, $\mathrm{L}$.

\section{AMELANCHIER}

alnifolia, Nutt.

Canadensis, T, \& G. var. (?) oblongifolia, T. \& G. var. (?) oligocarpa, T. \& G. var. rotundifolia, T. \& G. 


\section{CHAIM झRHODOS}

erecta, Bunge. var. Nuttallii, T. \& G.

\section{CRATAFGUS}

coccinea, $\mathrm{L}$.

Crus-galli, L.

Douglasii, Lindl.

Oxyacantha, $L$.

rivularis, Nutt.

subvillosa, Schrad.

tomentosa, L.

var. punctata, Gray.

var. pyrifolia, Gray.

\section{DALIBARDA}

repens, L.

(Rubus Dalibarda, L.)

\section{DRYAS}

octopetala, L.

var. Drummond i, Watson.

(D. Drummondii, Hook.)

var. integrifolia, Ch. \& Sch.

\section{FRAGARIA}

Chilensis, Duchesne.

var. Scouleri, Hook. vesca, $\mathrm{L}$.

Virginiana, Duchesne.

\section{GEUIM}

album, Gmel.

calthifolium, Menzies.

glaciale, Fisch.

macrophyllum, Willd.

rivale, $\mathrm{L}$.

Rossii, Ser.

var. humile, T. \& G. strictum, Ait.

triflorum, Pursh.

vernum, T. \& G.

Virginianum, L.
GILLENIA

trifoliata, Mœench.

\section{NEILLIA}

opulifolia, Benth. \& Hook. var. mollis, Brew. \& Wats.

\section{NUTPALIA}

cerasiformis, T. \& G.

\section{PIRUS}

Acuparia, L. (?)

Americana, DC.

arbutifolia, L. f.

var. melanocarpa, Hook.

malus, $L$.

rivularis, Dougl.

sambucifolia, Cham. \& Schl.

\section{POTENTILLA}

Anserina, L.

var. grandis, Lehm.

var. Grœnlandica, Tratt.

argentea, $\mathrm{L}$.

arguta, Pursh.

biflora, Willd.

Canadensis, $\mathrm{L}$.

var. simplex, T. \& G. dissecta, Pursh.

var. glaucophylla, Lehm.

var. multisecta, Watson.

var. pinnatisecta, Watson.

effusa, Dougl.

emarginata, Pursh.

fragiformis, Willd.

var. villosa, Reg. \& Tiling. frigida, Villars.

fruticosa, $\mathrm{L}$.

gelida, C. A. Mever.

glandulosa, Lindl.

gracilis, Dougl.

var. fastigiata, Watson.

var. flabelliformis, T. \& G. 
var. rigida, Watson.

Hippiana, Lehm.

var. pulcherrima, Watson.

Hookeriana, Lehm.

humifusa, Nutt.

maculata, Pourret.

nemoralis, Nestler.

nivea, $\mathrm{L}$.

var. dissecta, Watson.

var. Vahliana, Seem.

Norvegica, L.

var. hirsuta, T. \& G.

palustris, Scop.

Pennsylvanica, $\mathrm{L}$.

var. bipinnatifida, T. \& G.

var. glabrata, Watson.

var. strigosa, Pursh.

pilosa, Willd.

Plattensis, Nutt.

pulchella, R. Br.

rivalis, Nutt. var. millegrana,

Watson.

supina, $\mathrm{L}$.

tridentata, Sol.

\section{POTERIUM}

annuum, Nutt.

Canadense, Benth, \& Hook. officinale, Benth. \& Hook.

Sanguisorba, $L$.

Sitchense, Watson.

\section{PRUNUS}

Americana, Marsh.

var. mollis, 'T. \& G.

demissa, Walp.

emarginata, Walp.

var: mollis, Brewer.

maritima, Wangenh.

Pennsylvanica, L. f.

purnila, L.

serotina, Ehrh.

Virginiana, L.

\section{PURSHIA}

tridentata, DC.

\section{ROSA}

acicularis, Lindl.

Arkansana, Porter.

(acicularis, Lindl, Var. Bourgeaniana, Crepin

blanda, Ait.

Californica, Cham. \& Schl.

Carolina, L.

Fendleri, Crepin.

( $\mathrm{R}$ parviflora, Ehrh.)

gymnocarpa, Nutt.

humilis, Marsh.

luciảa, Ehrh.

micrantha, Smith.

nitida, Willd.

Nutkana, Presl.

pisocarpa, Gray.

pruinosa, Baker.

rubiginosa, $L$.

Sayi, Schw.

(R. blanda, Ait. var. setigera, Crepin.)

setigera, Michx.

Woodsii, Lindl.

\section{RUBUS}

articus, L.

var. grandiflorus, Ledeb.

Canadensis, $\mathbf{L}$.

Chamamorus, $I_{\text {s. }}$.

hispidus, $\mathrm{L}$.

var. setosus, T. \& G.

leucodermis, Dougl.

neglectus, Peck.

Nutkanus, Moc.

nivalis, Dougl.

occidentalis, $\mathrm{I}$.

odoratus, L.

pedatus, Smith.

spectabilis, Pursh.

stellatus, Smith.

strigosus, Michx.

triflorus, Richard.

ursinus, Cham. \& Schlecht.

villosus, Ait. 
var. frondosus, Torr.

var. humifusus, T. \& G.

\section{SIBBALDIA}

procumbens, L.

\section{SPIR円A}

Aruncus, L.

betulifolia, Pallas. var. rosea, Gray.

Douglasii, Hook.

var. Menziesii, Presl.

discolor, Pursh., var. ariæfolia,

Watson.

pectinata, T. \& G.

salicifolia, L.

tomentosa, $\mathrm{L}$.

\section{WALDSTEINIA}

fragarioides, Tratt.

\section{SAXIFRAGACEA.}

\section{BOYKINIA}

occidentalis, T. \& G.

Richardsoni, Gray.

\section{CHRYSOSPLENIUIM}

alternifolium, L.

Americanum, Schw.

\section{HEUCHERA}

Americana, L.

cylindrica, Dougl.

var. alpina, Watson.

(S. Hallii, Graý.)

glabra, Willd.

hispida, Pursh. micrantha, Dougl.

parvifolia, Nutt.

\section{LEPTARRHENA}

pyrolifolia, R. Br.

\section{MITELLA}

Breweri, Gray.

caulescens, Nutt,

diphylla, L.

nuda, $\mathrm{L}$.

var. (M. prostrata, Michx.)

pentandra, Hook.

trifida, Gray.

\section{PARNASSIA}

Caroliniana, Michx.

fimbriata, Koenig.

Kotzebuei, Cham. \& Schl

palustris, L.

parviflora, DC.

\section{PHILADELPHUS}

Gordonianus, Lindl.

Lewisii, Pursh.

\section{RIBES}

aureum, Pursh.

bracteosum, Dougl.

cereum, Dougl.

Cynosbati, L.

divaricatum, Dougl.

var. irriguum, Gray.

floridum, L'Her.

Hudsonianum, Richard.

var. (R. petiolare, Dougl.)

lacustre, Poir.

var. molle, Gray.

var: parvulum, Gray.

laxiflorum, Pursh.

Lobbii, Gray.

oxyacanthoides, L.

prostratum, L'Her.

rotundifolium, Michx.

rubrum, L., var. subglandulosum,

Maxim.

sanguineum, Pursh.

setosum, Lindl.

viscosissimum, Pursh. 


\section{SAXIFRAGA}

adscendens, L.

azoides, $\mathrm{L}$.

Aizoon, Jacq.

bronchialis, L.

var. cherlerioides, Engler.

cæspitosa, L.

var. uniflora, Hook.

cernua, L.

debilis, Engelm.

Eschscholtzii, Sternb.

exilis, Stephan.

flagellaris, Willd.

hieracifolia, Waldst. \& Kit.

Hirculus, L.

integrifolia, Hook.

Jamesii, Torr.

leucanthemifolia, Michx., var.

Brunoniana, T. \& G.

var. ferruginea, T. \& G.

Lyallii, Engler.

Mertensiana, Bong.

(S. heterantha, Hook.)

nivalis, $\mathrm{L}$.

nudicaulis, Don.

occidentalis, Gray.

oppositifolia, L.

Pennsylvanica, L.

punctata, L.

var. acutidentata, Engler.

ranunculifolia, Hook.

reflexa, Hook.

rivularis, $\mathrm{L}$.

var. hyperborea, Hook.

var. Laurentiana, Engler.

serpyllifolia, Pursh.

sileniflora, Sternb.

stellaris, L.

var. comosa, Poir. tricuspidata, Retz.

Virginiensis, Michx.

\section{TELLIMA}

grandiflora, R. Br.

parviflora, Hook.

tenella, Walp,
TIARELIA

cordifolia, L.

laciniata, Hook.

trifoliata, L.

unifoliata, Hook.

\section{TOLIMIEA}

Menziesii, T. \& G.

\section{CRASSULACEAE.}

\section{PENTHORUM}

sedoides, L.

\section{SEDUM}

acre, $L$.

Douglasii, Hook.

obtusatum, Gray.

rhodanthum, Gray.

Rhodiola, DC.

spathulifolium, Hook

stenopetalum, Pursh.

Telephium, $L$.

ternatum, Michx.

\section{DROSERACEA.}

\section{DROSERA}

Anglica, Hudson.

intermedia, Drev. \& Hayne., var.

Americana, DC. linearis, Goldie. rotundifolia, L.

\section{HAMAMELACEAE.}

HAIMAMELIS

Virginiana, L. HALORAGEA.

\section{HIPPURIS}

maritima, Hellenius. 
montana, Ledeb. vulgaris, $\mathrm{L}$.

\section{MYRIOPHYLLUM}

alterniflorum, DC.

heterophyllum, Michx.

spicatum, L.

tenellum, Bigel.

verticillatum, $\mathrm{L}$.

\section{PROSERPINACA}

palustris, $\mathrm{L}$.

\section{CALLITRICHACEA.}

\section{CALLITRICHE}

autumnalis, L.

Bolanderi, Heglhim.

verna, $\mathrm{L}$.

\section{MELASTOMACEA.}

\section{RHEXIA}

Virginica, L.

\section{LYTHRACEA.}

\section{LYTHRUM}

alatum, Pursh.

Salicaria, L.

\section{NESAFA}

verticillata, HBK.

\section{ONAGRACEA.}

\section{BOISDUVAIIA}

densiflora, Watson.

glabella, Walp.
CIRCA A

alpina, $\mathrm{L}$.

Lutetiana, L.

Pacifica, Asch. \& Mag.

\section{CLARKIA}

pụlchella, Pursh.

\section{EPILOBIUIM}

affine, Bong.

alpinum, L.

var. nutans, Lehm.

coloratum, Muhl.

Franciscanum, Barbey.

latifolium, L.

luteum, Pursh.

minutum, Lindl.

var. folisum, T. \& G.

molle, Torr.

Oreganum, Hauskn.

origanifolium, Lam.

palustre, L.

var. lineare, Gray.

paniculatum, Nutt.

roseum, Schreb.

spicatum, Lam.

(E. angustifolium, L.) tetragonum, $\mathrm{L}$.

\section{GAURA}

biennis, L.

coccinea, Nutt.

\section{GODETIA}

amoena, Lilja.

hispidula, Wat.

epilobioides, Watson.

quadrivulnera, Spach.

\section{IUDWIGIA}

alternifolia, L.

palustris, Ell. 


\section{ENOTHERA}

albicaulis, Nutt.

biennis, L.

var. grandiflora, Lindl.

var. hirsutissima, Gray.

var. muricata, Lindl

breviflora, T. \& G.

cæspitosa, Nutt.

fruticosa, L.

pumila, L.

(C. chrysantha, Mx.)

serrulata, Nutt., var. Douglasii,

T. \& G.

sinuata, L.

strigulosa, T. \& G.

var: pubens, Watson.

triloba, Nutt.

\section{LOASACEA.}

\section{MENTZELIA}

albicaulis, Dougl.

lævicaulis, T. \& G.

ornata, T. \& G.

\section{CUCURBITACEAE.}

\section{ECHINOCYSTIS}

lobata, T. \& G.

\section{SICYOS}

angulatus, L.

\section{CACTACEAE.}

\section{MAMILLARIA}

vivipara, Haworth.

\section{OPUNTIA}

fragilis Haworth.

Missouriensis, DC.

Rafinesquii, Engelm.
FICOIDEA.

MOLLUGO

verticillata, $\mathrm{L}$.

\section{UMBELLIFERA.}

\section{ÆTHUSA.}

Cynapium, $L$.

\section{ANGELICA}

Dawsoni, Watson. genuflexa, Nutt. lucida, $\mathrm{L}$. Lyallii, Watson.

\section{ANTHUSCUS}

vulgaris, Pers.

\section{ARCHANGELICA}

atropurpurea, Hoffm.

Gmelini, DC.

hirsuta, T. \& G.

\section{ARCHEMORA}

rigida, DC.

\section{BERULA}

angustifolia, Koch.

\section{BUPLEURUIM}

ranunculoides, L.

rotundifolium, $L$.

\section{CARUIM}

Carui, $L$.

Gairdneri, Benth. \& Hook.

Oreganum, Watson. 


\section{CAUCALIS}

nodosa, Huds.

\section{CHÆROPHYLLUM}

procumbens, Crantz.

\section{CICUTA}

bulbifera, L.

Californica, Gray.

maculata, L.

virosa, $\mathrm{L}$.

\section{CONIUM}

maculatum, $L$.

\section{CRANTZIA}

lineata, Nutt.

\section{CRYPTOT ÆNIA}

Canadensis, DC.

\section{CYMOPTERUS}

glomeratus, Raf. terebinthinus, T. \& G.

\section{DAUCUS}

Carota, $L$.

pusillus, Michx., var. microphyllus, T. \& G.

\section{ERIGENIA}

bulbosa, Nutt.

\section{FERULA}

dissoluta, Watson. multifida, Gray.

\section{GLYCOSIMA}

occidentale, Nutt.

\section{HERACLEUM}

lanatum, Michx.

\section{HYDROCOTYLE}

Americana, L. prolifera, Kellogg.

\section{LIGUSTICUIM}

actæifolium, Michx.

apiifolium, Benth. \& Hook.

Scoticum, L.

\section{MUSENIUIM}

divaricatum, Nutt.

var. Hookeri, T. \& G.

tenuifolium, Nutt.

\section{GNANTHE}

sarmentosa, Presl.

\section{OSIMORRHIZA}

brevistylis, DC.

longistylis, DC. nuda, Torr.

\section{PEUCEDNAUIM}

ambiguum, Nutt. fœniculaceum, Nutt.

leiocarpum, Nutt. microcarpum, Nutt.

Martindalii, C. \& R., var. angustatum, C.\& R.

Sandbergii, C. \& R. sativum, Benth. \& Hook. triternatum, Nutt. utriculatum, Nutt. villosuin, Nutt.

\section{PHELLOPTERUS}

littoralis, Schmidt.

\section{PIMPINELLA}

integerrima, Benth. \& Hook.

\section{SANICULA}

arctopoides, Hook. \& Arn. bipinnatifida, Dougl. 
Canadensis, L.

Howellii, C. \& R.

Marilandica, M.

Menziesii, Hook. \& Arn.

Nevadensis, Wat.

\section{SELINUIM}

Benthami, Watson.

Canadense, Michx.

Dawsoni, C. \& R.

Pacificum, Watson.

\section{SIUIM}

cicutæfolium, Gmel.

\section{THASPIUM}

aureuin, Nutt.

barbinode, Nutt.

trifoliatum, Gray.

\section{ARALIACEA.}

\section{ARAIIA}

hispida, Vent.

nudicaulis, $\mathrm{L}$.

quinquefolia, Dec. \& Planch.

racemosa, $\mathrm{L}$.

trifolia, Dec. \& Planch.

\section{FATSIA}

horrida, Benth. \& Hook.

\section{CORNACEA.}

\section{CORNUS}

alternifolia, L. f.

asperifolia, Michx.

Canadensis, $\mathrm{L}$.

circinata, L'Her.

florida, L.

Nuttallii, Audubon.

paniculata, L'Her.

pubescens, Nutt.

sericea, L.

stolonifera, Michx.
Suecica, L.

Unalaskensis, Ledeb.

NYSSA

multiflora, Wangenh.

CAPRIFOLIACEA.

ADOXA

Moschatellina, L.

DIERVILLA

trifida, Mœench.

\section{LINN画A}

borealis, Gronov. var. longiflora, Torr.

LONICERA

cærulea, L. var. villosa, T. \& G.

ciliata, Muhl.

ciliosa, Poir.

glauca, Hill.

hirsuta, Eaton.

hispidula, Dougl.

var. vacillans, Gray. involucrata, Banks. oblongifolia, Hook. Sullivantii, Gray. Tartarica, $L$. Utahensis, Watson.

\section{SAMBUCUS}

Canadensis, L. glauca, Nutt. melanocarpa, Gray. racemosa, $\mathrm{L}$. var. arborescens, T. \& G.

\section{SYMPHORICARPOS}

mollis, Nutt.

var. acutus, Gray. occidentalis, Hook. racemosus, Michx. var. pauciflorus, Robbins. 


\section{TRIOSTEUM}

perfoliatum, L.

\section{VIBURNUM}

acerifolium, $\mathrm{L}$.

cassinoides, $\mathrm{L}$.

(S. nudum, L. var. cassinoides, T. \& G.)

dentatum, L.

lantanoides, Michx.

Lentago, $\mathrm{L}$.

Opulus, L.

pauciflorum, Pylaie.

pubescens, Pursh.

\section{RUBIACEA.}

\section{GALIUIM}

Aparine, L.

var. Vaillantii, Koch.

asprellum, Michx.

boreale, L.

(G. rubioides, L.)

circæzans, Michx.

Kamtschaticum, Steller.

lanceolatum, Torr.

Mollugo, $L$.

pilosum, Ait.

tricorne, With.

trifidum, L.

(var. tinctorium, T. \& G.)

var. bifolium, Macoun.

var. latifolium, Torr.

var. pusillum, Gray.

triflorum, Michx.

verum, $L$.

\section{HOUSTONIA}

cæerulea, L.

purpurea, L. var. ciliolata, Gray.

var. longif $\lrcorner$ lia, Gray.

\section{MITCHELLA}

repens, $\mathrm{L}$.

\section{SHERARDIA}

arvensis, $L$.

\section{VALERIANACEA.}

\section{VALERIANA}

capitata, Pall.

edulis, Nutt.

Sitchensis, Bong.

(V. capitata, Willd, var. Hookeri, T. \& G.)

sylvatica, Banks.

(V. dioica, L. var. sylvatica, Rich.)

(V. dioica, L. var. uliginosa, T. \& G.)

\section{VALERIANELLA}

anomala, Gray. congesta, Lindl.

(Plectritis, congesta, DC.) macrocera, Gray. olitoria, Poll.

(Fedia olitoria, Vahl.) samolifolia, Gray.

\section{DIPSACACEA.}

\section{DIPSACUS}

sylvestris, Mill.

\section{COMPOSITA.}

\section{ACHILLEA}

Millefolium, L.

var. lanata, Koch.

var. nigrescens, L.

multiflora, Hook.

Ptarmica, L.

\section{ACTINELLA}

acaulis, Nutt.

Richardsonii, Nutt. 


\section{ACTINOMERIS}

squarrosa, Nutt.

\section{ADENOCAULON}

bicolor, Hook.

\section{AIMBROSIA}

artemisiæfolia, L. psilostachya, DC.

trifida, L.

var. integrifolia, T. \& G.

\section{ANAPHALIS}

margaritacea, B. \& H.

\section{ANTENNARIA}

alpina, Gærtn.

Carpathica, R. Br.

var. pulcherrima, Hook.

dimorpha, T. \& G.

dioica, Gærtn.

var parviflora, T. \& G.

var. rosea, Eaton.

luzuloides, T. \& G.

plantaginifolia, Hook.

racemosa, Hook.

\section{ANTHEMIS}

arvensis, $L$.

Cotula, $L$.

(Maruta Cotula, DC.)

tinctoria, $L$.

\section{APARGIDIUM}

boreale, T. \& G.

\section{APLOPAPPUS}

acaulis, Gray.

(A. acaulis, var. glabratus, Eaton.)

Brandegei, Gray.

lanceolatus, T. \& G.

var. Vaseyi, Parry.
Nuttallii, T. \& G. spinulosus, DC. uniflorus, T. \& G.

\section{ARCTIUIM}

Lappa, $L$. var. minus, Gray. var. tomentosum, Gray.

\section{ARNICA}

alpina, Olin.

amplexicaulis, Nutt.

Chamissonis, Less. cordifolia, Hook.

foliosa, Nutt.

latifolia, Bong. obtusifolia, Less.

Unalaschensis, Less.

\section{ARTEMISIA}

borealis, Pall. var. Wormskioldii, Bess. cana, Pursh.

Canadensis, Michx. caudata, Michx. discolor, Dougl. var. incompta, Gray. dracunculoides, Pursh. glauca, Pall. Lindleyana, Bess. longifolia, Nutt. Ludoviciana, Nutt. tridentata, Nutt. trifida, Nutt.

\section{ASTER}

acuminatus, Michx. adscendens, Lindl. alpinus, $\mathrm{L}$. amplus, Lindl. angustus, 'T. \& G. azureus, Lindl. campestris, Nutt. canescens, Pursh. commutatus, Gray. 
(A. multiflorus, var. commutatus, T. \& G.) conspicuus, Lindl. cordifolius, $\mathrm{L}$. corymbosus, Ait. diffusus, Ait. Douglasii, Lindl. dumosus, L. Engelmanni, Gray. ericoides, L.

var. villosus, T. \& G. falcatus, Lindl. foliaceus, Lindl.

var. Eatoni, Gray. var. frondeus, Gray. var. pubescens, Gray. Fremonti, Gray. junceus, Ait. lævis, L. linariifolius, $\mathrm{L}$. Lindleyanus, T. \& G. var. ciliolatus, Gray. longifolius, Lam. macrophyllus, $\mathrm{L}$. Menziesii, Lindl. modestus, Lindl.

(A. mutatus, T. \& G.)

(A. Sayii, Gray.)

multiflorus, Ait.

var, stricticaulis, T. \& G. nemoralis, Ait.

Novæ-Angliæ, L.

Novi-Belgii, L.

var. lævigatus, Gray.

(A. lævigatus, Lam.)

var. litoreus, Gray.

occidentalis, Nutt.

var. intermedius, Gray.

Oreganus, Nutt.

(A. elegans, T. \& G.) paniculatus, Lam.

patens, Ait.

pauciflorus, Nutt.

peregrinus, Pursh. prenanthoides, Muhl. ptarmicoides, T. \& G. var. lutescens, Gray.

(A. lutescens, T. \& G.) puniceus, L.

var. lævicaulis, Gray.

(var. firmus, T. \& G.)

var. lucidulus, Gray.

pygmæus, Lindl.

radula, Ait.

var. strictus, Gray.

radulinus, Gray.

sagittifolius, Willd.

salicifolius, (Lam.?) Ait. sericeus, Vent.

Sibiricus, L.

(A. montanus, Rich.)

var. giganteus, Gray. spathulatus, Lindl. subulatus, Michx.

(A. linifolius, L.) tanacetifolius, HBK. tardiflorus. L.

Tradescanti, L. . umbellatus, Mill. var. pubens, Gray. var. villosus, Gr.

undulatus, $\mathrm{L}$. vimineus, Lam.

var. foliolosus, Gray.

\section{BALSAMORRHIZA}

deltoidea, Nutt. hirsuta, Nutt. sagittata, Nutt.

\section{BELIIS}

perennis, $L$.

\section{BIDENS}

Beckii, Torr.

bullata, L. (?)

cernua, $\mathrm{L}$.

chrysanthemoides, Michx. connata, Muhl.

frondosa, $\mathrm{L}$. 


\section{BIGELOVIA}

Douglasii, Gray. graveolens, Gray.

(B. graveolens, var. hololeuca, Gray.)

\section{BOLTONIA}

latisquama, Gray,

\section{CACALIA}

atriplicifolia, L. tuberosa, Nutt.

\section{CARDUUS}

nutans, $L$.

crispus, $L$.

\section{CENTAUREA}

benedicta, $L$.

Calcitrapa, L. .

Cyanus, $L$.

Jacea, $L$.

Melitensis, $L$.

nigra, $L$.

\section{CH FNACTIS}

Douglasii, Hook. \& Arn.

\section{CHRYSANTHEMUIM}

arcticum, L.

Balsamita, $L$.

bipinnatum, L.

integrifolium, Richards,

Leucanthemum, $L$.

Parthenium, Pers.

segetum, $L$.

\section{CHRYSOPSIS}

villosa, Nutt.

var. hispida, Gray.

\section{CICHORIUM}

Intybus, $L$.

\section{CNICUS}

altissimus, Willd., var. discolor, Gray.

arvensis, Hoffm.

Drummondii, Gray.

var. acaulescens, Gray. edulis, Gray.

eriocephalus, Gray.

foliosus, Gray.

Hookerianus, Gray.

Kamtschaticus, Maxim.

lanceolatus, Hoffm.

muticus, Pursh.

Pitcheri, Torr.

pumilus, Torr.

undulatus, Gray.

\section{COREOPSIS}

discoidea, T. \& G.

lanceolata, $\mathrm{L}$.

palmata, Nutt.

tinctoria, Nutt.

trichosperma, Michx., var. tenuiloba, Gray.

tripteris, $\mathrm{L}$.

verticillata, $\mathrm{L}$.

\section{COTULA}

coronopifolia, $L$.

\section{CREPIS}

acuminata, Nutt.

biennis, $L$.

elegans, Hook.

glauca, T. \& G.

intermedia, Gray.

nana, Richards.

occidentalis, Nutt.

var. crinita, Gray.

var. Nevadensis, Kellogg. runcinata, T. \& G. virens, $L$.

\section{DYSODIA}

chrysanthemoides, Lag. 


\section{ECHIN ACEA}

angustifolia, DC.

\section{ERECHTITES}

hieracifolia, Raf.

\section{ERIGERON}

acris, $\mathrm{L}$.

(E. alpinus, Linn.)

var. debilis, Gray.

var. Drobachensis, Blytt.

annuus, Pers.

armeriæfolius, Turcz.

Bellidiastrum, Nutt.

bellidifolius, Muhl.

cæspitosus, Nutt.

Canadensis, L.

compositus, Pursh.

var. discoideus, Gray.

(var. glabratus, Macoun.)

concinnus, T. \& G.

corymbosus, Nutt. eriocephalus, J. Vahl. filifolius, Nutt.

flagellaris, Gray. glabellus, Nutt.

var. asperus, T. \& G.

var. mollis, Gray.

var. pubescens, Hook.

grandiflorus, Hook.

hyssopifolius, Michx.

lanatus, Hook.

macranthus, Nutt.

peucephyllus, Gray.

Philadelphicus, L.

poliospermus, Gray.

pumilus, Nutt.

radicatus, Hook.

salsuginosus, Gray.

speciosus, DC.

strigosus, Muhl.

uniflorus, $\mathrm{L}$.

\section{ERIOPHYLLUM}

cæspitosum, Dougl.

var. integrifolium, Gray

var. leucophyllum, Gray.
EPUATORIUM

ageratoides, L. f.

perfoliatum, $L$.

purpureum, L.,

var. maculatum, Darl.

rotundifolium, $\mathrm{L}$.

\section{FRANSERIA}

bipinnatifida, Nutt.

Chamissonis, Less.

Hookeriana, Nutt.

\section{GAILLARDIA}

aristata, Pursh.

\section{GNAPHALIUIM}

decurrens, Ives.

microcephalum, Nutt.

Norvegicum Gunner.

palustre, Nutt.

polycephalum, Michx.

purpureum, L.

Sprengelii, Hook. \& Arn.

supinum, Vill.

sylvaticum, $\mathrm{L}$.

uliginosum, L.

\section{GRINDELIA}

hirsutula, Hook. \& Arn. integrifolia, DC.

(G. stricta, DC.)

nana, Nutt.

var. discoidea, Gray. squarrosa, Dunal.

\section{GUTIERREZIA}

Euthamiæ, T. \& G.

\section{HELENIUIM}

autumnale, $\mathrm{L}$.

var. grandiflorum, Gray.

\section{HELIANTHUS}

annuus, L. 
decapetalus, $\mathrm{L}$.

divaricatus, $\mathrm{L}$.

doronicoides, Lam.

giganteus, $\mathrm{L}$.

Maximiliani, Schrad.

Nuttallii, T. \& G.

parviflorus, Bernh.

petiolaris, Nutt.

pumilus, Nutt.

rigidus, Desf.

strumosus, L.

tuberosus, L.

\section{HELIOPSIS}

lævis, Pers.

scabra, Dunal.

\section{HIERACIUM}

albiflorum, Hook.

aurantiacum, $L$.

Canadense, Michx.

gracile, Hook.

var. detonsum, Gray.

Gronovii, L.

longipilum, Torr.

murorum, $L$.

paniculatum, L.

peletarium, Mer.

Pilosella. $L$.

scabrum, Michx.

Scouleri, Hook.

triste, Cham.

umbellatum, L.

venosum, $\mathrm{L}$.

vulgatum, Fries.

\section{HYMENOPAPPUS}

filifolius, Hook.

\section{HYPOCHCERIS}

glabra, $L$.

radicata, $L$.

\section{INULA}

Helenium, $L$.
IVA

axillaris, Pursh.

xanthiifolia, Nutt.

JAUIMEA

carnosa, Gray.

\section{KRIGIA}

amplexicaulis, Nutt.

Virginica, Willd.

\section{LACTUCA}

Canadensis, L.

Floridana, Gærtn.

hirsuta, Muhl.

integrifolia, Bigel.

leucophæa, Gray.

pulchella, DC.

Scariola, $L$.

sativa, $L$.

\section{LAMPSANA}

communis, $L$.

\section{LAYIA}

glandulosa, Hook. \& Arn.

\section{LEONTODON}

autumnalis, $L$.

hirtus, $L$.

\section{LEPACHYS}

columnaris, T. \& G.

(Rudbeckia columnaris, Pursh ) var. pulcherrima, T. \& G.

\section{IIATRIS}

cylindracea, Michx. punctata, Hook. scariosa, Willd. spictata, Willd. 


\section{IUINA}

hypoleuca, Benth.

\section{IYGODESIMIA}

juncea. Don. rostrata, Gray.

\section{MIADIA.}

filipes, Gray. glomerata, Hook.

Nuttallii, Gray. sativa, Molina. var. racemosa, Gray. (M. dissitiflora, T. \& G.)

\section{IMATRICARIA}

discoidea, DC. inodora, $L$.

var. nana, Hook.

\section{MICROSERIS}

Bigelovii, Gray. nutans. Gray.

\section{MIKANIA}

scandens, Gray.

\section{ONOPORDON}

acanthium, $L$.

\section{PETASITES}

frigida, Fries.

palmata, Gray.

sagittata, Gray.

\section{PICRIS}

echinoides, $L$.

hieracioides, L., var. Japonica, Regel.

\section{POLYIMNIA}

Canadensis, $\mathrm{L}$.
PRENANTHES

alata, Gray.

var. sagittata, Gray.

alba, L.

altissima, $\mathrm{L}$.

racemosa. Michx.

serpentaria, Pursh.

var. nana, Gray.

\section{PSILOCARPHUS}

elatior, Gray. tenellus, Nutt.

\section{RUDBECKIA}

hirta, L.

laciniata, L,

\section{SA USSUREA}

alpina, DC. var. Ledebouri, Gray.

\section{SENECIO}

amplectens, Gray. aureus, $\mathrm{L}$.

var. Balsamitæ, T. \& G. var. borealis, T. \& G. var. compactus, Gray. var. croceus, Gray. var. discoideus, Hook. var. lanceolatus, Oaks. var. obovatus, T. \& G. var. subnudus, Gray. canus, Hook. eremophilus, Richards. fastigiatus, Nutt. Fremonti, T. \& G. frigidus, Less, Hookeri, T. \& G. hydrophilus, Nutt. integerrimus, Nutt. Jacoboea, Linn. lugens, Richards. var. exaltatus, Gray. var. foliosus, Gray. 
megacephalus, Nutt. palustris, Hook. petræus, Klatt. Pseudo-Arnica, Less. resedifolius, Less.

var. Columbiensis, Gray sylvaticus, $L$.

triangularis, Hook. viscosus, $L$. vulgaris, $L$.

\section{SERICOCARPUS}

solidagineus, Nees.

\section{SILPHIUIM}

perfoliatum, L.

terebinthinaceum, Jacq.

\section{SILYBUM}

Marianum, Gortn.

\section{SOLIDAGO}

arguta, Ait.

bicolor, L.

var. concolor, T. \& G.

var. lanata, Gray.

crsia, L.

var. axillaris. Gray.

Canadensis, $\mathrm{L}$.

var. procera, T. \& G.

var. scabra, T. \& G.

confertiflora, DC.

elongata, Nutt.

Houghtoni, T. \& G.

humilis, Pursh.

var. nana, Gray.

juncea, Ait.

lanceolata, L.

latifolia, $\mathrm{L}$.

lepida, DC.

macrophylla, Pursh.

Missouriensis, Nutt.

var. montana, Gray.

multiradiata, Ait.

var. scopulorum, Gray. neglecta, T. \& G.

nemoralis, Ait.

var. incana, Gray.

occidentalis, Nutt.

odora, Ait.

Ohioensis, Riddell.

patula, Muhl.

puberula, Nutt.

Riddellii, Frank.

rigida, $\mathrm{L}$.

rugosa, Mill.

sempervirens, L.

serotina, Ait.

var. gigantea, Gray.

speoiosa, Nutt.

squarrosa, Muhl.

Terræ-Novæ, T. \& G.

uliginosa, Nutt.

Virgaurea, L., var. alpina, Big.

\section{SONCHUS}

arvensis, $L$.

asper, Vill.

oleraceus, $L$.

\section{STEPHANOIMERIA}

minor, Nutt.

TANACETUM

Huronense, Nutt.

vulgare, $L$.

\section{TARAXACUM} officinale, Weber.

var. alpinum, Koch.

var. glaucescens. Koch.

var. lividum, Koch.

var. scopulorum, Gray.

TETRADYMIA canescens, DC.

\section{TOWNSENDIA}

Parryi, Eaton, var. alpina, Gray

(T. florifer, Gray.)

sericea, Hook. 


\section{TRAGOPOGON}

porrifolius, $L$. pratensis, $L$.

\section{TROXIMON}

aurantiacum, Hook.

cuspidatum, Pursh.

glaucum, Nutt.

var. dasycephalum, T. \& G.

var. parviflorum, Gray.

var. taraxacifolium, Gray. gracilens, Gray.

grandiflorum, Gray.

heterophyllum, Greene.

humile, Gray.

laciniatum, Gray.

\section{TUSSILAGO}

Farfara, $L$,

\section{VERNONIA}

altissima. Nutt.

Noveboracensis, Willd.

\section{WYETHIA}

amplexicaulis, Nutt.

\section{XANTHIUM}

Canadense, Mill.

var. echinatum, Gray.

spinosum, $L$.

\section{LOBELIACEA.}

\section{LOBELIA}

cardinalis, $\mathrm{L}$.

Dortmanna, L.

inflata, L.

Kalmii, L.

spicata, Lam.

var. hirtella, Gray.

syphilitica, L.

\section{CAMPANULACEA.}

CAMPANULA.

Americana, L. aparinoides, Pursh. lasiocarpa. Cham. pilosa, Pall. rapunculoides, $L$. rotundifolia, L.

var. arctica, Lange.

var. Alaskana, Gray.

Scouleri, Hook. uniflora, L.

\section{HETEROCODON}

rariflorum, Nutt.

\section{SPECULARIA}

biflora, Gray. perfoliata, A. DC.

\section{ERICACEA.}

\section{ARCTOSTAPHYLOS}

alpina, Spreng.

tomentosa, Dougl.

Uva-ursi, Spreng.

\section{ANDROMEDA}

ligustrina, Muhl.

polifolia, L.

\section{ALLOTROPA}

virgata, T. \& G.

\section{ARBUTUS}

Menziesii, Pursh.

\section{BRYANTHUS}

Aleuticus, Gray. empetriformis, Gray. var. intermedius, Gray. 
(B. Grahamii, Hook.) glanduliflorus, Gray. taxifolius, Gray.

\section{CALLUNA}

vulgaris, Salisb.

\section{CASSANDRA}

calyculata, Don.

\section{CASSIOPE}

hypnoides, Don. lycopodioides, Don. Mertensiana, Don. Stelleriana, DC. tetragona, Don.

\section{CHIMAPHILA}

maculata, Pursh.

Menziesii, Spreng. umbellata, Nutt.

\section{CHIOGENES}

hispidula, T. \& G.

\section{CLADOTHAMNUS}

pyrolæflorus, Bong.

\section{EPIG 画A}

repens, $\mathrm{L}$.

\section{GAULTHERIA}

Myrsinites, Hook. ovatifolia, Gray. procumbens, L. Shallon, Pursh.

\section{GAYLUSSACIA}

dumosa, T. \& G. resinosa, T. \& G.

\section{KALMIA}

angustifolia, L. glauca, Ait.

var. microphylla, Hook. latifolia, $\mathrm{L}$.

\section{LEDUI}

glandulosum, Nutt. latifolium, Ait. palustre, L. var. dilatatum, Wahl.

\section{LOISELEURIA} procumbens, Desv.

\section{IMENZIESIA}

ferruginea, Smith. glabella, Gray.

\section{MONESES}

uniflora, Gray.

\section{MONOTROPA}

fimbriata, Gray. Hypopitys, L.

(Hypopitys lanuginosa, Nutt. uniflora, $\mathrm{L}$.

\section{PTEROSPORA}

andromedea, Nutt.

\section{PYROLA}

aphylla, Smith.

chlorantha, Swartz.

var. occidentalis, Gray.

elliptica, Nutt.

minor, $\mathrm{L}$.

picta, Smith.

rotundifolia, L.

var. asarifolia, Hook.

var. bracteata, Gray.

var. incarnata, DC.

var. pumila, Hook.

var. uliginosa, Gray.

secunda, L.

var. pumila, Gray. 


\section{RHODODENDRON}

albiflorum, Hook.

Californicum, Hook.

(R. macrophyllum, Don.)

Kamtschaticum, Pall.

Lapponicum, Wahl.

maximum, $\mathrm{L}$.

nudiflorum, Torr.

Rhodora, Gmelin.

viscosum, Torr.

\section{VACCINIUIM}

cæspitosum, Michx.

var. cuneifolium, Nutt.

Canadense, Kalm.

corymbosum, L.

var. amœenum, Gray.

var. atrococcum, Gray.

var. pallidum, Gray.

macrocarpon, Ait.

(Oxycoccus macrocarpus Pursh.)

myrtilloides, Hook.

var. rigidum, Hook.

Myrtillus, L.

var. microphyllum, dook occidentale, Gray.

ovalifolium, Smith.

ovatum, Pursh.

Oxycoccus, $\mathrm{L}$.

(Oxycoccus vulgaris, Pursh.)

var. intermedium, Gray. parvifolium, Smith.

Pennsylvanicum, Lam.

var. augustifolium, Gray.

salicinum, Cham.

stamineum, L.

uliginosum, L.

var. mucronatum, Herder. vacillans, Solander.

Vitis-Idæa, L.

\section{DIAPENSIACEA.}

\section{DIAPENSIA}

Lapponica, I.

\section{PLUMBAGINACEA.}

\section{ARIMERIA}

vulgaris, Willd.

\section{STATICE}

Limonium, L., var. Caroliniana, Gray.

\section{PRIMULACEA.}

\section{ANAGALLIS}

arvensis, $L$.

\section{ANDROSACE}

Chamæjasme, Host. occidentalis, Pursh. septentrionalis, L.

\section{CENTUNCULUS}

minimus, $\mathrm{L}$.

\section{DODECATHEON}

Media, L.

Jeffreyi, Moore.

Hendersoni, Gray.

frigidum, Cham. \& Schl.

\section{DOUGLASIA}

arctica, Hook.

nivalis, Lindl.

\section{GLAUX}

maritima, L.

\section{LYSIMACHIA}

nummularia, $L$. punctata, $L$. quadrifolia, $\mathrm{L}$. stricta, Ait, thyrsiflora, L. 


\section{PRIMULA}

borealis, Duby.

cuneifolia, Ledeb.

Egaliksensis, Hornem.

farinosa, $\mathrm{L}$.

Mistassinica, Mic'ıx.

nivalis, Pall.

officinalis, Linn.

Sibirica, Jacq.

vulgaris, Huds.

\section{SAMOLUS}

Valerandi, L., var. Americanus, Gray.

\section{STEIRONEMA}

ciliatum, Raf.

lanceolatum, Gray.

longifolium, Gray.

\section{TRIENTALIS}

Americana, Pursh.

Europæa, L.

var. arctica, Ledeb.

var. latifolia, Torr.

\section{OLEACEA.}

\section{FRAXINUS}

Americana, L.

Oregana, Nutt.

pubescens, Lam.

quadrangulata, Michx.

sambucifolia, Lam.

var. Berlandieriana, Gray.

\section{LIGUSTRUIM}

vulgare, $L$.

\section{APOCYNACEA.}

\section{APOCYNUM}

androsæmifolium, L.

(var. incanum, A. DC.) (var. glabrum, Macoun.)

var. pumilum, Gray.

cannabinum, $\mathrm{L}$.

(var. pubescens, Macoun.)

(var. glaberrimum, DC.)

(var. hypericfoliun, Gray.)

\section{ASCLEPIADACEA.}

\section{ACERATES}

viridiflora, Ell.

var. lanceolata, Gray.

var. linearis, Gray.

\section{ASCLEPIAS}

Cornuti, Decaisne.

incarnata, $\mathrm{L}$.

ovalifolia, Decaisne.

phytolaccoides, Pursh.

purpurascens, L.

quadrifolia, L.

speciosa, Torr.

tuberosa, L.

verticillata, $\mathrm{L}$.

\section{GENTIANACEA.}

\section{BARTONIA}

tenella, Muhl.

\section{FRASERA}

Carolinensis, Walt,

\section{GENTIANA}

affinis, Griseb.

(G. puberula, Mx.)

alba, Muhl.

Amarella, L., var. acuta, Hk. f

var. stricta, Watson.

var. tenuis, Gray.

Andrewsii, Griseb. arctophila, Griseb.

aurea, $\mathrm{L}$. 
auriculata, Pall.

calycosa, Griseb.

crinita, Froel.

Douglasiana, Bong.

Forwoodii, Gray.

(G. affinis, Griseb.)

frigida, Hænke.

glauca, Pall.

humilis, Stev.

linearis, Frol.

var. lanceolata, Gray.

nivalis, $\mathrm{L}$.

Oregana, Engelm.

platypetala, Griseb.

propinqua, Richards.

prostrata, Hænke.

quinqueflora, Lam.

Saponaria, L.

sceptrum, Griseb.

serrata, Gunner.

tenella, Rottb.

ventricosa, Griseb.

\section{HALENIA}

deflexa, Griseb.

var. Brentoniana, Gray.

\section{LIMNANTHEMUMI}

lacunosum, Griseb.

\section{IMENY ANTHES}

Crista-galli, Menzies.

trifoliata, L.

\section{PLEUROGYNE}

Carinthiaca, Griseb, var. pusilla, Gray.

rotata, Griseb.

\section{SWERTIA}

perennis, L., var. obtusa, Griseb.

\section{POLEMONIACA.}

\section{GILIA}

achilleæfolia, Benth.

aggregata, Spreng.

capitata, Dougl.

coronopifolia, Pers.

grandiflora, Gray.

gracilis, Hook.

(Collomia gracilis, Doug].)

heterophylla, Dougl.

(Collomia heterophylla, Hook.)

inconspicua, Dougl.'

intertexta, Steud.

linearis, Gray.

(Collomia linearis, Nutt.)

liniflora, Benth., var. pharnaceoides, Gray.

minutiflora, Benth.

squarrosa, Hook. \& Arn.

tenella, Benth.

\section{PHLOX}

canescens, T. \& G.

divaricata, $\mathrm{L}$.

Douglasii, Hook. var. diffusa, Gray.

Hoodii, Richards.

linearifolia, Gray.

longifolia, Nutt.

pilosa, L.

Richardsonii, Hook.

Sibirica, L.

speciosa, Pursh.

subulata, L.

\section{POLEIMONIUM}

cæruleum, L.

var. acutiflorum, Ledeb.

confertum, Gray.

humile, Willd.

var. pulchellum, Gray. micranthum, Benth. 


\section{HYDROPHYLLACEAE.}

\section{FLLISIA}

Nyctelea, L.

\section{HYDROPHYLLUM}

appendiculatum, Michx.

Canadense $\mathrm{L}$.

capitatum, Dougl.

Virginicum, L.

\section{NEMOPHILA}

Menziesii, Hook. \& Arn.

parviflora, Dougl.

\section{PHACELIA}

circinata, Jacq. $\mathbf{f}$.

Franklinii, Gray.

Menziesii, Torr.

sericea, Gray.

var. Lyallii, Gray.

\section{ROMANZOFFIA}

Sitchensis, Bong.

Unalaschkensis, Cham.

\section{BORRAGINACEA.}

\section{AMSINCKIA}

intermedia, Fisch. \& Meyer.

lycopsoides, Lehm.

var. bracteosa, Gray.

\section{BORRAGO}

officinalis, $L$.

\section{CYNOGLOSSUIM}

ciliatum, Dougl.

grande, Dougl.

occidentale, Gray.

officinale, $L$.

Virginicum, L.
ECHINOSPERIMUM

brachycentrum, Ledeb., var. brachystylum Gray.

deflexum, Lehm, var. Americanum, Gray.

diffusum, Lehm.

floribundum, Lehm.

Lappula, Lehm.

Redowskii, Lehm., var. cupulatum, Gray. var. occidentale. Watson.

Virginicum, Lehm.

\section{ECHIUIM}

vulgare, $L$.

\section{HELIOTROPIUM}

Curassavicum, L.

KRYNITZKIA (Eritrichium.)

Californica, Gray.

Chorisiana, Gray.

circumscissa, Gray.

crassisepala, Gray.

glomerata, Gray.

leiocarpa, Fisch. \& Meyer.

leucophæa, Gray.

plebeia, Gray.

Scouleri, Gray.

(Eritrichium fulvum, A. DC.)

\section{LITHOSPERMUIM}

angustifolium, Michx.

arvense, $L$.

canescens, Lehm.

hirtum, Lehm.

latifolium, Michx.

officinale, $L$.

pilosum, Nutt.

\section{LYCOPSIS}

arvensis, $L$. 


\section{MERTENSIA}

lanceulata, DC.

maritima, Don.

oblongifolia, Don.

paniculata, Don.

Sibirica, Don.

var. Drummondii, Gray.

Virginica, DC.

\section{MYOSOTIS}

arvensis, Hoffm.

laxa, Lehm.

palustris, With.

sylvatica, Hoffim., var. alpestris, Koch.

verna, Nutt.

var. macrosperma, Chapm.

OIMPHALODES (Eritrichium.) nana, Gray., var. aretioides, Gray.

\section{ONOSMIODIUIM}

Carolinianum, DC.

var. molle, Gray.

Virginianum, DC.

\section{PECTOCARYA}

penicillata, A. DC.

\section{PLAGIOBOTHRYS}

(Eritrichium.)

tenellus, Gray.

Torreyi, Gray.

\section{SYMPHYTUIM}

officinale, $L$.

\section{CONVOLVULACEA.}

\section{CONVOLVULUS}

arvensis, $L$.

sepium, L., var. Americanus, Sims.

var. maritima, Macoun. var. repens, Gray.

Soldanella, L.

spithamæus, L.

\section{CUSCUTA}

arvensis, Beyrich. compacta, Juss. epilinum, Weihe. Gronovii, Willd. salina, Engelm. tenuiflora, Engelm. Trifolii, Bab.

\section{IPOMEAA}

pandurata, Meyer.

1. urqurea, Lam.

SOLANACEA.

\section{DATURA}

Stramonium, $I$.

Tatula, $L$.

\section{HYOSCY AMUS}

niger, $L$.

\section{LYCIUIM}

vulgare, Dunal.

\section{LYCOPERSICUM}

esculentum, Mill.

\section{NICANDRA}

physaloides, Goertn.

\section{NICOTIANA}

attenuata, Torr. rustica, $L$.

PETUNIA

parviflora, Juss. 


\section{PHYSALIS}

grandiflora, Hook.

lanceolata, Michx.

Peruviana, $L$.

pubescens, $\mathrm{L}$.

Virginiana, Mill.

var. ambigua, Gray.

\section{SOLANUM}

Carolinense, L.

Dulcamara, $L$.

nigrum, $\mathrm{L}$.

rostratum, Dunal.

triflorum, Nutt.

\section{SCROPHULARIACEA.}

\section{ANTIRRHINUIM}

Orontium, $L$.

\section{BARTSIA}

alpina, L.

Odontites, Huds.

\section{CASTILLEIA}

coccinea, Spreng.

miniata, Dougl.

pallida, Kunth.

var. septentrionalis, Gray. parviflora, Bong. sessiliflora, Pursh.

\section{CHELONE}

glabra, L.

nemorosa, Dougl.

\section{COLLINSIA}

grandiflora, Dougl.

var. pusilla, Gray.

parviflora, Dougl.

\section{DIGITALIS}

purpurea, $L$.

\section{EUPHRASIA}

officinalis, L.

var. Tartarica, Benth.

\section{GERARDIA}

aspera, Dougl.

flava, L.

pedicularia, L.

purpurea, L.

var. paupercula, Gray. quercifolia, Pursh. tenuifolia, Vahl.

var. asperula, Gray.

\section{GRATIOLA}

aurea, Muhl. ebracteata, Benth.

Virginiana, L.

\section{ILYSANTHES}

gratioloides, Benth.

\section{LIMOSELLA}

aquatica, $\mathrm{L}$.

var. tenuifolia, Hoffm.

\section{LINARIA}

Canadensis, Dumont.

Cymbalaria, Mill.

Elatine, Mill.

minor, Desf.

vulgaris, $L$.

\section{IMELAIMPYRUIM}

Americanum, Michx.

\section{MIMULUS}

alsinoides, Benth.

floribundus, Dougl.

glabratus, HBK., var. Jamesii,

Gray.

(M. Jamesii, T. \& G.)

Lewisii, Pursh.

luteus, $\mathrm{L}$. 
var. alpinus, Gray.

var. depauperatus, Gray. moschatus, Dougl. nasutus, Greene. ringens, $\mathrm{L}$.

\section{ORTHOCARPUS}

attenuatus, Gray. bracteosus, Benth. castilleioides, Benth. hispidus, Benth. luteus, Nutt. pusillus, Benth. tenuifolius, Benth.

\section{PEDICULARIS}

bracteosa, Benth.

Canadensis, L. capitata, Adams. Chamissonis, Stev. contorta, Benth. euphrasioides, Stephan.

flammea, L.

Furbishiæ, Watson.

Groelandica, Retz. hirsuta, L.

lanceolata, Michx. Langsdorffii, Fisch. var. lanata, Gray.

Lapponica, L.

Menziesii, Benth.

palustris, L., var. Wlassoviana, Bunge.

pedicellata, Bunge.

racemosa, Dougl.

Sudetica, Willd.

versicolor, Wahl.

verticillata, $L$.

\section{PENTSTEMON}

accuminatus, Dougl.

albidus, Nutt.

(P. cristatus, Nutt.)

confertus, Dougl.

var. cæruleo-purpureus, Gr. deustus, Dougl.

diffusus, Dougl.

frutescens, Lamb.

glaucus, Graham.

gracilis, Nutt.

humilis, Nutt.

Menziesii, Hook.

var. Scouleri, Gray.

ovatus, Dougl.

pubescens, Solander.

triphyllus, Dougl.

venustus, Dougl.

\section{RHINANTHUS}

Crista-galli, L.

\section{SCROPHULARIA}

Californica, Cham.

lanceolata, Pursh.

nodosa, L., var. Marilandica, Gray.

\section{SYNTHYRIS}

rubra, Benth.

\section{VERBASCUIM}

Blattaria, $L$.

Lychnitis, $L$.

Thapsus, $L$.

\section{VERONICA}

agrestis, $L$.

alpina, $\mathrm{L}$.

Americana, Schwein.

Anagallis, L.

arvensis, $L$.

Buxbaumii, Tenore.

Chamoedrys, L.

Kamtchatica, L. f.

officinalis, $\cdot \mathrm{L}$.

peregrina, $\mathrm{L}$.

scutellata, L.

var. pubescens, Macoun.

serpyllifolia, L.

Stelleri, Pall.

Virginica, L. 


\section{OROBANCHACEA.}

\section{APHYLLON}

comosum. Gray.

fasciculatum, Gray.

Ludovicianum, Gray. pinetorum, Gray. uniflorum, Gray.

\section{BOSCHNIAKIA}

glabra, C. A. Meyer.

Hookeri, Walp.

\section{CONOPHOLIS}

Americana, Wallr.

\section{EPIPHEGUS}

Virginiana, Bart.

\section{LENTIBULARIACEA.}

\section{PINGUICULA}

alpina, $\mathrm{L}$.

villosa, $\mathrm{L}$.

vulgaris, $\mathrm{L}$.

\section{UTRICULARIA}

clandestina, Nutt. cornuta, Michx.

gibba, $\mathrm{L}$.

inflata, Walt.

intermedia, Hayne.

minor, $\mathrm{L}$.

resupinata, B. D. Greene.

vulgaris, $L$.

\section{BIGNONIACEAE.}

\section{TECOMA}

radicans, Juss.
ACANTHACEA.

\section{DIANTHERA}

Americana, L.

\section{SELAGINACEA.}

\section{LAGOTIS}

glauca, Gærtn.

(Gymnanda Gmelini, Cham. \& Schl.)

(Gymnandra Stelleri, Cham. \& Schl.)

VERBENACEA.

\section{PHRYMA}

Leptostachya, L.

\section{VERBENA}

angustifolia, Michx.

bracteosa, Michx.

hastata, L.

urticæfolia, L.

\section{LABIATEA.}

AJUGA

reptans, $L$.

BALLOTA

nigra, $L$.

BLEPHILIA

hirsuta, Benth.

BRUNELIA

vulgaris, $\mathrm{L}$. 


\section{CALAMINTHA}

clinopodium, Benth.

Nuttallii, Benth.

\section{COLLINSONIA}

Canadensis, L.

\section{DRACOCEPHAIUM}

parviflorum Nutt.

\section{GALEOPSIS}

Ladanum, L.

Tetrahit, $L$.

\section{HEDEOIMA}

hispida, Pursh.

pulegioides, Pers.

\section{HYSSOPUS}

officinalis, $L$.

\section{ISANTHUS}

cæruleus, Michx

\section{LAMIUM}

album, $L$.

amplexicaule, $L$.

purpureum, L.

\section{LEONURUS}

Cardiaca, $L$.

\section{IOPHANTHUS}

anisatus, Benth.

nepetoides, Benth.

scrophulariæfolius, Bənth.

\section{LYCOPUS}

lucidus, Turcz., var. Americanus, Gray. sinuatus, Ell.

Virginicus, L.
MARRUBIUM

vulgare, $L$.

\section{MELISSA}

officinalis, $L$.

\section{MENTHA}

aquatica, $L$.

arvensis, $L$.

Canadensis, L.

var. glabrata, Benth.

piperita, $L$.

sativa, $L$.

viridis, $L$.

\section{MICROMERIA}

Douglasii, Benth.

\section{MONARDA}

clinopodia, L.

didyma, $\mathrm{L}$.

fistulosa, $\mathrm{L}$.

var. mollis, Benth.

punctata, L.

\section{NEPETA}

Cataria, L.

Glechoma, Benth.

\section{ORIGANUIM}

vulgare, $L$.

\section{PHYSOSTEGIA}

parviflora, Nutt.

Virginiana, Benth.

\section{PYCNANTHEMUIM}

incanum, Michx.

lanceolatum, Pursh.

muticum, Pers., var. pilosum, Gray. 
SATUREIA

hortensis, $L$.

\section{SCUTELLARIA}

angustifolia, Pursh.

canescens, Nutt.

galericulata, $\mathbf{L}$.

lateriflora, $\mathrm{L}$.

parvula, Michx.

\section{STACHYS}

aspera, Michx.

ciliata, Dougl.

var. pubens, Gray.

palustris, L.

\section{TEUCRIUM}

Botrys, $L$,

Canadense, L.

occidentale, Gray.

\section{THYMUS}

Serpyllum, $L$.

\section{PLANTAGINACEA.}

\section{LITTORELLA}

lacustris, $\mathrm{L}$.

\section{PLANTAGO}

Bigelovii, Gray.

cordata, Lam.

decipiens, Barneoud.

eriopoda, Torr.

lanceolata. $L$.

macrocarpa, Cham. \& Schl.

major, L.

var. Asiatica, Decaisne.

var. bracteata, Macoun.

var. minima, Decaisne.

maritima, $\mathrm{L}$.

media, $L$.

Patagonica, Jacq., var. aristata, Gray. var. gnaphalioides, Gray.

var. spinulosa, Gray. pusilla, Nutt.

Rugelii, Decaisne.

\section{NYCTAGINACEAE.}

\section{ABRONIA}

latifolia, Esch.

umbellata, Lam.

\section{OXYBAPHUS}

angustifolius, Sweet. hirsutus, Sweet. nyctagineus, Sweet.

\section{AMARANTACEAE.}

\section{ACNIDA}

ruscocarpa, Michx. tuberculata. Gray.

\section{AMARANTUS}

albus, L.

blitoides, Watson.

hypochondriacus, $L$. paniculatus, $L$.

retroflexus, $L$.

\section{CHENOPODIACEA.}

\section{ATRIPLEX}

Alaskensis, Watson.

arenaria, Nutt.

argentea, Nutt.

Endolepis, Watson.

Gmelini, C. A. Meyer.

Nuttallii, Watson.

patula, L., var. hastata, Gray.

var. littoralis, Gray.

var. subspictata, Watson.

rosea, $L$.

zosteræfolia, Watson. 


\section{AXYRIS}

amarantioides, $L$.

\section{CHENOPODIUIM}

album, $L$. var. viride.

ambrosioides, $L$.

var anthelminticum, Gray.

Bonus-Henricus, L.

Botrys, $L$.

capitatum, Watson.

Fremonti, Watson.

glaucum, L.

humile, Hook.

(C. rubrum, L., var. humile, Moq.)

hybridum, L.

leptophyllum, Nutt.

var. subglabrum, Watson.

rubrum, L.

urbicum, $L$.

\section{CORISPERIMUIM}

hyssopifolium, L.

\section{CYCLOLOIMA}

platyphyllum, Moq.

\section{EUROTIA}

lanata, Moq.

\section{KOCHIA}

scoparia, $L$.

\section{IMONOLEPIS}

chenopodioides, Moq.

\section{SALICORNIA}

ambigua, Michx.

herbaca, L.

mucronata, Bigel.

\section{SAISOLA}

Kali, L.

\section{SARCOBATUS}

vermiculatus, Torr.

\section{SUADA}

depressa, Watson.

var. erecta, Watson.

linearis, Torr., var. ramosa, Watson.

PHYTOLACCACEA.

\section{PHYTOLACCA}

decandra, L.

\section{POLYGONACEA.}

\section{ERIOGONUIM}

androsaceum, Benth.

flavum, Nutt.

heracleoides, Nutt.

multiceps, Nees.

ovalifolium, Nutt.

umbellatum, Torr.

FAGOPYRUM

esculentum, Monch.

KOENIGIA

Islandica, L.

OXYRIA

digyna, Campdera.

\section{POLYGONUII}

acre, HBK.

amphibium, L.

arifolium, L.

articulatum, $\mathrm{L}$.

aviculare, $\mathrm{L}$.

Bistorta, L.

Careyi, Olney.

cilinode, Michx. 
coarctatum, Dougl.

Convolvulus, $L$.

dumetorum, L., var. scand $n$ s, Gray.

erectum, L.

Hartwrightii, Gray.

Hydropiper, $L$.

hydropiperoides, Michx.

imbricatum, Nutt.

incarnatum, Ell.

intermedium, Nutt.

lapathifolium, Ait.

maritimum, L.

minimum, Watson.

Muhlenbergii, Watson.

nodosum, Pers.

orientale, $L$.

Paronychia, Cham. \& Schl.

Pennsylvanicum, L.

Persicaria, $L$.

polymorphum, Ledeb

ramosissimum, Michx.

sagittatum, L.

Tartaricum, $L$.

tenue, Michx.

var. latifolium, Engelm.

Virginianum, L.

viviparum, $\mathrm{L}$.

\section{RUMEX}

Acetosa, $L$.

Acetosella, L.

Britannicus, L.

(R. orbiculatus, Gray.)

crispus, $\mathrm{L}$.

maritimus, $\mathrm{L}$.

obtusifolius, $L$.

occidentalis, Watson.

Patientia, $L$.

paucifolius, Nutt.

salicifolius, Weinm.

sanguineus, $L$.

venosus, Pursh.

verticillatus, $\mathrm{L}$.
ARISTOLOCHIACEA.

ASARUIM

Canadense, L.

caudatum, Lindl.

\section{PIPERACEA.}

\section{SAURURUS}

cernuus, L.

\section{LAURACEA.}

\section{IINDERA}

Benzoin, Meisn.

SASSAFRAS

officinale, Nees.

THYMELEACEAE.

DAPHNE

Mezereum, $L$.

\section{DIRCA}

palustris, L.

ELAEAGNACEA.

EL ÆAGNUS

argentea, Pursh.

\section{SHEPHERDIA}

argentea, Nutt.

Canadensis, Nutt.

\section{LORANTHACEA.}

\section{ARCEUTHOBIUM}

Americanum, Nutt.

robustum, Engelm.

vaginatum, Eichler. 


\section{SANTALACEA.}

\section{COMANDRA}

livida, Richards.

pallida, A. DC.

umbellata, Nutt.

\section{EUPHORBIACEA.}

\section{ACALYPHA}

Virginica, L.

\section{EUPHORBIA}

commutata, Engelm.

corollata, L.

Cyparissias, $L$.

glyptosperma, Engelm.

Helioscopia, $L$.

hypericifolia, L.

maculata, $\mathrm{L}$.

obtusata, Pursh.

Peplus, L.

platyphylla, $L$.

polygonifolia, L.

serpens, HBK.

serpyllifolia, Pers.

\section{MERCURIALIS}

annua, $L$.

\section{URTICACEA.}

\section{BFHMERIA}

cylindrica, Willd.

\section{CANNABIS}

sativa, $L$.

\section{CELTIS}

occidentalis, $\mathrm{L}$.

\section{HUIMULUS}

Lupulus, L.

\section{IAPORTEA}

Canadensis, Gaudich.

\section{IMORUS}

alba, $L$.

rubra, $\mathrm{L}$.

\section{PARIETARIA}

Pennsylvanica, Muhl.

\section{PILEA}

pumila, Gray.

\section{ULIMUS}

Americana, L. fulva, Michx.

racemosa, Thomas.

\section{URTICA}

dioica, $L$.

gracilis, Ait.

holosericea, Nutt.

Lyallii, Watson.

urens, $L$.

\section{PLATANACEA.}

\section{PLATANUS}

occidentalis, $\mathrm{L}$.

\section{JUGLANDACEAE.}

\section{CARYA}

alba, Nutt.

amara, Nutt.

porcina, Nutt.

tomentosa, Nutt.

\section{JUGLANS}

cinerea, $\mathrm{L}$. nigra, $\mathrm{L}$. 


\section{MYRICACEA.}

\section{MYRICA}

asplenifolia, Endl.

Californica, Cham.

cerifera, L.

Gale, L.

\section{BETULACEA.}

\section{ALNUS}

incanus, Willd.

var. virescens, Watson.

rhombifolia, Nutt.

rubra, Bong.

viridis, DC.

\section{BETULA}

alba, var. populifolia, Spach.

glandulosa, Michx.

lenta, $\mathrm{L}$.

lutea, Michx. f.

nigra, $\mathrm{L}$.

occidentalis, Hook. papyrifera, Michx. pumila, L.

\section{CUPULIFERA.}

\section{CARPINUS}

Caroliniana, Walt.

\section{CASTANEA}

vesca, $\mathrm{L}$, var. Americana, Mx.

\section{CORYLUS}

Americana, Walt.

rostrata, Ait.

var. Californica, A. DC.

\section{FAGUS}

fermginea, Ait.
OSTRYA

Virginica, Willd.

\section{QUERCUS}

alba, L.

bicolor, Willd.

coccinea, Willd.

Garryana, Dougl.

macrocarpa, Michx.

obtusiloba, Michx.

palustris, Du R.ji.

prinoides, Willd.

Prinus, L.

rubra, $\mathrm{L}$.

tinctoria, Bartr.

\section{SALICACEA.}

\section{POPULUS}

angustifolia, James.

balsamifera, L.

var. candicans, Gray. grandidentata, Michx. monilifera, Ait. tremuloides, Michx. trichocarpa, T. \& G.

\section{SALIX}

adenophylla, Hook. alba, L., var. coerulea, Koch. amygdaloides, And. arctica, Pall.

var. petræa, And. argyrocarpa, And. balsamifera, Barratt. Barclayi, And.

var. latiuscula, Aud. Barrattiana, Hook. candida, Willd.

Chamissonis, And. chlorophylla, And. cordata, Muhl.

var. angustata, And.

var. Mackenziana, Hook. 
desertorum Rich.

discolor, Muhl.

var. eriocephala, And.

Drummondiana, Barratt.

flavescens, Nutt.

var. Scouleriana, Bebb.

var. tenuijulis, And.

fulcrata, var. subglauca, And.

glauca, L.

var. villosa, And.

glaucophylla, Bebb.

herbacea, L.

Hookeriana, Barratt.

humilima, And.

humilis, Muhl.

lanata, $\mathrm{L}$.

var. Macouniana, Bebb. N. var.

lasiandra, Benth.

var. lancifolia Bebb.

longifolia, Muhl.

var. argyrophylla, And.

lucida, Muhl.

macrocarpa, Nutt.

myrtilloides, $\mathrm{L}$.

nigra, Marsh.

Novæ-Angliæ, And.,var. myrtillifolia, And.

var. pseudo-cordata, And.

var. pseudo-myrsinites, And.

orbicularis, And.

ovalifolia, Traut.

Pallasii, Anders., var. crassijulis, Anders.

var. diplodyctya, Anders.

petiolaris, Smith.

var. gracilis, And.

phlebophylla, And.

phylicoides, And.

polaris, Wahl.

prolixa, And.

reticulata, $\mathrm{L}$.

var. nivalis, And.

Richardsoniana, Hook.

rostrata, Rich.

sericea, Marsh.

sessilifolia, Nutt.
Sitchensis, Sanson.

var. angustifolia, Bebb.

speciosa, Hook. \& Arn.

tristis, Ait.

Uva-ursi, Pursh.

vestita, Pursh.

viminalis, $L$.

\section{EMPETRACEA.}

\section{COREIMA}

Conradii, Torr.

EMPETRUM

nigrum, L.

CERATOPHYLLACEA.

CER ATOPHYLLUM

demersum, L.

\section{CONIFERA.}

\section{ABIES}

amabilis, Forbes.

balsamea, Marshall.

grandis, Lindl.

subalpina, Engelm.

\section{JUNIPERUS}

communis, L.

var. alpina, Gaud.

occidentalis, Hook.

Sabina, L., var. procumbens, $\mathrm{Ph}$.

Virginiana, L.

\section{LARIX}

Americana, Michx.

Lyallii, Parlat.

occidentalis, Nutt. 
PICEA

alba, Link.

Engelmanni, Engelm.

nigra, $\mathrm{L}$.

Sitchensis, Carr.

\section{PINUS}

albicaulis, Engelm.

Banksiana, Lambert.

contorta, Dougl.

flexilis, James.

monticola, Dougl.

Murrayana, Balf.

ponderosa, Dougl., var. scopulorum, Engelm.

resinosa, Ait.

rigida, Mill.

Strobus, L.

\section{PSEUDOTSUGA}

Douglasii, Carr.

\section{TAXUS}

baccata, L., var. Canadensis, Gray.

brevifolia, Nutt.

\section{THUYA}

excelsa, Bong.

gigantea, Nutt.

occidentalis, L.

sphæroidea, Spreng.

\section{TSUGA}

Canadensis, Carr.

Mertensiana, Carr.

Pattoniana, Engelm.

\section{HYDROCHARIDACEAE.}

\section{ELODEA}

Canadense, Planch.
VALLISNERIA

spiralis, L.

ORCHIDACEA.

\section{APLECTRUM}

hiemale, Nutt.

\section{ARETHUSA}

bulbosa, L.

\section{CALOPOGON}

pulchellus, R. Br.

\section{CALYPSO}

borealis, Salisb.

CORALLORHIZA

innata, $\mathrm{R}$. Br.

Mertensiana, Bong.

multiflora, Nutt.

odontorhiza, Nutt.

striata, Lindl.

\section{CYPRIPEDIUM}

acaule, Ait.

arietinum, $\mathrm{R}$. Br.

montanum, Dougl.

parviflorum, Salisb. passerinum, Rich. pubescens, Willd. spectabile, Swartz.

\section{EPIPACTIS}

gigantea, Dougl.

\section{GOODYERA}

Menziesii, Lindl. pubescens, R. Br. repens, R. Br. 


\section{HABENARIA}

blephariglottis, Hook.

bracteata, R. Br.

ciliaris, $\mathrm{R}$. Br.

dilatata, Gray.

elegans, Bolander.

fimbriata, R. Br.

gracilis, Watson.

Hookeri, Torr.

var. oblongifolia, Paine.

hyperborea, R. Br.

lacera, R. Br.

leucophæa, Gray.

leucostachys, Watson.

Menziesii, Lindl. obtusata, Richards.

orbiculata, Torr.

psycodes, Gray.

sparsiflora, Watson.

tridentata, Gray.

Unalaschensis, Watson.

virescens, Spreng.

\section{IIPARIS}

Lœselii, Richards.

\section{LISTERA}

convallarioides, Nutt.

cordata, R. Br.

\section{MICROSTYLIS}

monophyllos, Lindl.

ophioglossoides, Nutt.

\section{ORCHIS}

rotundifolia, Pursh. spectabilis, L.

\section{POGONIA}

ophioglossoides, Nutt. pendula, Lindl. verticillata, Nutt.

\section{SPIR ANTHES}

cernua, Richard.

gracilis, Bigel.

latifolia, Torr.

Romanzoffiana, Cham.

\section{HAMODORACEAE.}

\section{ALETRIS}

farinosa, $\mathrm{L}$.

\section{IRIDACEA.}

\section{IRIS}

Hookeri, Penny.

lacustris, Nutt.

tenax, Dougl.

versicolor, L.

Virginica, L.

\section{SISYRINCHIUIM}

anceps, L.

Californicum, Ait. f. grandiflorum, Dougl.

mucronatum, Michx.

\section{AMARYLLIDACEA.}

\section{HYPOXIS}

erecta, L.

\section{DIOSCOREACEA.}

\section{DIOSCOR표}

villosa, Torr.

\section{LILIACEAE.}

\section{ALLIUM}

acuminatum, Hook.

Canadense, Kalm. 
cernuum, Roth.

Geyeri, Watson.

Nevii, Watson. reticulatum, Fras.

Schœenoprasum, L, stellatum, Fras. tricoccum, Ait.

Vancouverense, Macoun.

\section{ASPARAGUS}

officinalis, $L$.

\section{BRODI ÆA}

Douglasii, Watson. grandiflora, Smith. lactea, Watson.

\section{CALOCHORTUS}

elegans, Pursh., var. nanus, Wood.

macrocarpus, Dougl.

\section{CAMASSIA}

esculenta, Lindl.

Fraseri, Torr.

Leichtlinii, Watson.

\section{CHAM FLIRIUM}

Carolinianum, Willd.

\section{CLINTONIA}

borealis, Raf. uniflora, Kunth.

\section{ERYTHRONIUM}

albidum, Nutt.

Americanum, Smith. grandiflorum, Pursh.

var. (?) albiflorum, Hook.

var. (?) minus, Morren.

var. giganteum, Hook.

var. (?) Smithii, Hook. propullans, Gray.

\section{FRITILLARIA}

Kamtschatcensis, Ker.

lanceolata, Pursh. var. floribunda, Benth. var. gracilis, Watson:

pudica, Spreng.

\section{HEMEROCALLIS}

fulva, $L$.

\section{LILIUM}

Canadense, L.

Carolinianum, Michx..

Columbianum, Hanson.

Philadelphicum, L.

\section{ILOYDIA}

serotina, Reich.

\section{MAIANTHEMUM}

bifolium, DC., var. dilatatum, Wood.

Canadense, Desf,

\section{MEDEOLA}

Virginiana, L.

MELANTHIUM

Virginicum, L.

POLYGONATUIM

biflorum, L.

giganteum, Deitr.

\section{PROSARTES}

Hookeri, Torr. lanuginosa, Don. Menziesii, Don. Oregana, Watson. trachycarpa, Watson. 
SMILACINA

amplexicaulis, Nutt.

racemosa, Desf.

sessilifolia, Nutt.

stellata, Desf.

trifolia, Desf.

\section{SIMILAX}

herbacea, L

hispida, Muhl.

quadrangularis, Pursh.

STENANTHIUM

occidentale, Gray.

\section{SIREPTOPUS}

amplexifolius, DC.

roseus, Michx.

\section{TOFIELDIA}

borealis, Wahl. coccinea, Richards.

var. major, Hook.

glutinosa, Willd.

occidentalis, Watson.

\section{TRILLIUIM}

cernuum, L.

erectum, L., var. atropurpureum.

Hook.

var. album, Pursh.

var. declinatum, Gray.

vaa. ochroleucum, Hook.

erythrocarpurn, Michx.

grandiflorum, Salisb.

ovatum, Pursh.

\section{UVULARIA}

grandiflora, Smith.

perfoliata, $\mathrm{L}$ sessilifolia, $\mathrm{L}$.

\section{VERATRUIM}

viride, Ait.
XEROPHYLLUM

tenax, Nutt.

\section{ZYGADENUS}

elegans, Pursh.

paniculatus, Watson.

venenosus, Watson.

\section{PONTEDERIACEA.}

\section{HETERANTHERA}

graminea, Vahl.

\section{PONTEDERIA}

cordata, L.

var. angustifolia, Gray.

\section{XYRIDACEA}

\section{XYRIS}

flexuosa, Muhl., var. pusilla, Gray.

\section{JUNACEA.}

\section{JUNCUS}

acuminatus, Michx., var. legitimus, Engelm.

alpinus, Vill., var. insignis, Fr.

arcticus, Willd.

var. Sitchensis, Engelm.

articulatus, L.

Balticus, Dethard, var. littoralis, Engelm.

var. montanus, Engelm.

biglumis, $\mathrm{L}$.

bufonius, $\mathrm{L}$.

var. fasciculiflorus, Boiss.

Canadensis, J. Gay, var. coarctatus, Errgelm.

var. longecaudatus, Eng.

castaneus. Smith.

Drummondii, Mever. 
efftrsus, L.

var. brunneus, Engelm.

var. conglomeratus, Gray.

falcatus, E. Meyer.

filiformis, $\mathrm{L}$.

Gerardi, Lois.

Greenei, Oakes \& Tuckerm.

Lescurii, Bolander.

longistylis, Torr.

marginatus, Rostk., var. paucicapitatus, Engelm.

var. vulgaris. Engelm.

Mertensianus, Bong.

var paniculatus, Engelm.

militaris, Bigel.

Nevadensis, Watson.

nodosus, L., var. genuinus,

Engelm.

var. megacephalus, Torr.

Parryi, Engelm.

pelocarpus, E. Meyer.

var. (?) subtilis, Engelm. stygius, L.

supiniformis, Engelm.

tenuis, Willd.

var. congestus, Engelm.

var. secundus, Engelm.

trifidus, L.

triglumis, L.

Vaseyi, Engelm.

xiphioides, E. Mey., var. littoralis,

Engelm.

var. macranthus, Engelm.

var. montanus, Engelm.

var. triandrus, Engelm.

\section{LUZULA}

arcuata, Meyer.

campestris, Desv., var. como a,

Hook.

var. pallescens, Hook.

var. vulgaris, Hook.

comosa, Meyer.

var. congesta, Watson.

var. macrantha, Watson.

var. subsessilis, Watson. divaricata, Watson.

hyperborea, R. Br., var. major, Hook.

var. minor, Hook.

pilosa, Willd.

spadicea, DC., var. melanocarpa,

Meyer.

var. parviflora, Meyer.

var. subcongesta, Meyer.

spicata, Desv.

\section{TYPHACEA.}

\section{SPARGANIUIM}

affine, Schnitz.

androcladum, Morong.

eurycarpum, Engelm.

hyperboreum, Læst., var. Americanum, Beeby.

minimum, Bauhin.

simplex, Huds.

\section{TYPHA}

angustifolia, $\mathrm{L}$.

latifolia, $\mathrm{L}$.

\section{ARACEAE.}

\section{ACORUS}

Calamus, L.

ARISAFIMA

Dracontium, Schott. triphyllum. Torr.

\section{CALLA}

palustris, $\mathrm{L}$,

\section{LYSICHITON}

Kamtschatcense, Schott.

PELTANDRA

Virginica, Raf. 
SYMPLOCARPUS

fœtidus, Salisb.

\section{LEMNACEA.}

\section{LEMNA}

minor, $\mathrm{L}$.

polyrrhiza, $\mathrm{L}$.

trisulca, $\mathrm{L}$.

\section{WOLFFIA}

Brasiliensis, Weddell.

Columbiana, Karsten.

\section{ALISMACEAE.}

\section{ALISIMA}

Plantago, L., var. Americanum, Gray.

\section{DAMASONIUIM}

Californicum, Torr.

\section{ECHINODORUS}

parvulus, Engelm.

\section{SAGITTARIA}

calycina, Eng., var. spongiosa,

Engelm.

graminea, Pursh.

heterophylla, Pursh.

var. rigida, Engelm.

variabilis, Eng., var. angustifolia,

Engelm.

var. diversifolia, Engelm.

var. gracilis, Engelm.

var. hastata, Engelm.

var. latifolia, Engelm.

var. obtusa, Engelm.

var. pubescens, Engelm.

\section{NAIADACEAE.}

\section{IIL再A}

subulata, HBK.

\section{NAIAS}

flexilis, Rostk. \& Schmidt. var. robusta, Morong.

\section{PHYLLOSPADIX}

Scouleri, Hook.

\section{POTAMOGETON}

amplifolius, Tuckerm.

Claytonii, Tuckerm. gramineus, L.

var. graminifolius, Fries.

var. heterophyllus, Fries.

var. maximus, Morong.

hybridus, Michx.

lonchites, Tuckerm.

lucens, $\mathrm{L}$.

marinus, $\mathrm{L}$.

var. Macounii, Morong. mucronatus, Schrad. natans, $\mathrm{L}$.

var. prolixus, Koch. Oakesianus, Robbins. obtusifolius, M. \& K. pauciflorus, Pursh.

var. Niagarensis, Gray. pectinatus, $\mathrm{L}$. perfoliatus, $\mathrm{L}$.

var. lanceolatus, Robbins. prælongus, Wulf. pusillus, L.

var. panormitanus, Biv.

var. vulgaris, Fries.

Robbinsii, Oakes. rufescens, Schrad. rutilans, Wolfgang. Spirillus, Tuckerm. Vaseyi, Robbins. Zizii, M. \& K. zosterifolius, Schum.

\section{RUPPIA}

maritima, L. 
SCHEUCHZERIA

palustris, L.

\section{TRIGLOCHIN}

maritimum, $\mathrm{L}$. var. elatum, Gray. palustre, $\mathrm{L}$.

\section{ZANNICHELLIA}

palustris, $\mathrm{L}$.

\section{ZOSTERA.}

marina, $\mathrm{L}$.

\section{ERIOCAULONACEAE.}

\section{FRIOCAULON}

septangulare, With.

\section{CYPERACEA.}

\section{CAREX}

albata, Bailey.

acuta, $\mathrm{L}$.

var. prolixa, Hornem. adusta, Boott.

Alascana, Bœckl. alopecoidea, Tuckerm. alpina, Swartz. ambusta, Boott. aquatilis, Wahl.

var. epigeios, Læst. areta, Boott. arctata, Boott.

var. Faxoni, Bailey. arida, Schw. \& Torr. Assiniboinensis, W. Boott. athrostachya, Olney. atrata, L.

var. nigra. Boott.

var. ovata, Boott. atrofusca, Schk. aurea, Nutt.
Backii, Boott.

Barbaræ, Dew.

bicolor, All.

brizoides, L., var. nemoralis, Wimm.

bromoides, Schk.

Brongniartii, Kunth., var. densa, Bailey.

Buxbaumii, Wahl. cæspitosa, L.

var. filifolia, Boott.

canescens, $\mathrm{L}$.

var. alpicola, Wahl.

var. vulgaris, Bailey.

capillaris, $\mathrm{L}$.

capitata, L.

var. Krausei, Krantz.

cephaloidea, Boott.

cephalophora, Muhl.

var. angustifolia, Boott.

chordorhiza, Ehrh.

circinata, Meyer.

compacta, R. Br.

concinna, R. Br.

conoidea, Schk.

Crawei, Dew.

crinita, Lam.

var. gynandra, Sch. \& Torr.

cryptocarpa, Meyer.

debilis, Michx.

decidua, Boott.

Deweyana, Schw.

var. Bolanderi, W. Boott.

var. sparsiflora, Bailey.

digitalis, Willd.

dioica, $\mathrm{L}$.

disticha, Huds.

Douglasii, Boott.

eburnea, Boott.

echinata, Murr.

var. conferta, Bailey.

var. microstachys, Bœkl.

Emmonsii, Dew.

exilis, Dew.

festiva, Dew.

var. gracilis, Olney 
var. Haydeniana, W. Boott. filifolia, Nutt.

filiformis, $\mathrm{L}$.

flava, $\mathrm{L}$.

var. rectorostrata, Bailey.

flexilis, Rudge.

folliculata, $\mathrm{L}$.

formosa, Dew.

Franklinii, Boott.

fulva, Good. (?)

Gayana. Desv.

Geyeri, Boott.

glareosa, Wahl.

glauca, Scop.

Gmelini, Hook.

gracillima, Schw.

granularis, Muhl.

Grayii, Carey.

grisea, Wahl.

gynocrates, Wormsk.

heleonastes, Ehrh.

Hendersoni, Bailey.

heteroneura, W. Boott.

Hitchcockiana, Dew.

Hoodii, Boott.

Houghtonii, Torr.

hystricina, Willd.

incurva, Lightf.

intumescens, Rudge.

invisa, Bailey.

Jamesii, Torr.

lagopina, Wahl.

lanuginosa, Michx.

var. æmathorhyncha.

laxiflora, Lam.

var. intermedia, Boott.

var. latifolia, Boott.

var. patulifolia, Carey.

var. striatula, Carey.

leiocarpa, Meyer.

leiorhyncha, Meyer.

lenticularis, Michx.

leporina, L., var. Americana,

Olney.

Liddoni, Boott.

limosa, L. var. stygia, Bailey.

livida, Willd.

longirostris, Torr.

lurida, Wahl.

var. divergens, Bailey.

var. polystachya, Bailey.

Lyoni, Boott.

macrocephala, Willd.

Magellanica, Lam.

marcida, Boott.

maritima, Muller.

Meadii, Dew.

melanocarpa, Cham.

Mertensii, Prese.

Michauxiana, Bœekl.

microglochin, Wahl.

miliaris, Michx.

misandra, R. Br.

monile, Tuckerm.

Muhlenbergii, Schk.

muricata, L., var. confixa, Bailey.

var. gracilis, Boott.

nardina, Fries.

nigricans, Meyer.

Norvegica, Schk.

Novæ-Angliæ, Schw.

var. deflexa, Bailey.

var. Rossii, Bailey.

obesa, All., var. minor, Boott.

obtusata, Lilj.

Ederi, Retz.

oligocarpa, Schk.

oligosperma, Michx.

pallescens, $\mathrm{L}$.

panicea, $L$.

Parryana, Dew.

pauciflora, Lightf.

pedunculata, Muhl.

Pennsylvanica, Lam.

petricosa, Dew.

physocarpa, Presl.

pinguis, Bailey.

plantaginea, Lam.

platy phylla, Carey.

podocarpa, R. Br.

polytrichoides, Muhl. 
prasina, Wahl.

pratensis, Drej.

Pseudo-Cyperus, L.

var. comosa, W. Boott.

pubescens, Muhl.

Pyrenaica, Wahl.

Raeana, Boott.

rariflora, Smith.

Raynoldsii, Dew.

remota, $\mathrm{L}$.

retrocurva, Dew.

retrorsa, Schw.

var. Hartii, Carey.

Richardsonii, R. Br.

riparia, Curtis.

rosea, Schk.

var. radiata, Dew.

var. retroflexa, Torr.

rostrata, With.

var. utriculata, Bailey.

rotundata, Wahl.

rupestris, All.

salina, Wahl.

var. mutica, Wahl.

var. (?) robusta, Bailey .

Saskatschewana, Bœckl.

saxatilis, $\mathrm{L}$.

var. Grahami, Hook. \& Arn.

scabrata, Schw.

Schweinitzii, Dew.

scirpoidea, Michx.

scoparia, Schk.

siccata, Dew.

Sitchensis, Presc.

sparganioides, Muhl.

squarrosa, L.

stenophylla, Wahl,

Steudelii, Kunth.

stipata, Muhl.

straminea, Schk.

var. alata, Bailey.

var. aperta, Boott.

var. festucacea, Boott.

var. fœenea, Torr.

var. mixta, Bailey.

var. moniliformis, Tuckerm. var. tenera, Boott. stricta, Lam.

var. decora, Bailey. stylosa, Meyer.

subspathacea, Wormsk.

subulata, Michx.

sychnocephala, Carey.

tenella, Schk.

tentaculata, Muhl.

tenuiflora, Wahl.

teretiuscula, Good.

var. ramosa, Boott.

tetanica, Schk.

Tolmiei, Boott.

Torreyi, Tuckerm.

torta, Boott.

tribuloides, Wahl.

var. cristata, Bailey.

var. reducta, Bailey.

triceps, Michx.

trichocarpa, Muhl.

var. aristata, Bailey.

var. Deweyi, Bailey.

trisperma, Dew.

Tuckermani, Boott. umbellata, Schk.

var. brevirostris, Boott.

ursina, Dew.

vaginata, Tausch.

varia, Muhl.

vesicaria, $L$.

var. major, Boott. virescens, Muhl. vulgaris, Fries.

var. alpina, Boott.

var. hyperborea, Poott.

var. juncella, Fries.

vulpinoidea, Michx.

Willdenovii, Schk.

\section{CLADIUIM}

mariscoides, Torr.

\section{CYPERUS}

aristatus Rottb.

diandrus, Torr. 
var. castaneus, Torr. erythrorhizos, Muhl. esculentus, L. filiculmis, Vahl. flavescens, L. Schweinitzii, Torr. strigosus, L.

\section{DULICHIUIM}

spathaceum, Pers.

\section{ELFOCHARIS}

acicularis, R. Br. compressa, Sulliv. intermedia, Schultes. obtusa, Schultes. palustris, R. Br. var. calva, Gray. var. glaucescens, Gray. pauciflora, Watson. pygmæa, Torr. Robbinsii, Oakes.

var. occidentalis, Watson. tenuis, Schultes.

\section{ERIOPHORUIM}

alpinum, L. capitatum, Host. cyperinum, L.

var. laxus, Gray. gracile, Koch.

var. paucinervium, Engelm. lineatum, B. \& H. polystachyon, $\mathrm{L}$.

var: angustifolium, Gray. russeolum, Fries. vaginatum, $\mathrm{L}$. Virginicum, L.

var. album, Gray.

\section{KOBRESIA}

caricina, Willd. scirpina, Willd.

\section{RHYNCHOSPORA}

alba, Vahl.

capillacea, Torr.

fusca, R. \& S.

glomerata, Vahl

\section{SCIRPUS}

atrovirens, Muhl.

cæspitosus, L.

Clintonii, Gray.

fluviatilis, Gray.

lacustris, L.

var. occidentalis, Watson. maritimus, $\mathrm{L}$.

Nevadensis, Watson.

pungens, Vahl.

riparius, Spreng.

rufus, Schrad.

subterminalis, Torr.

sylvaticus, L., var. digynus, Bœeckl.

\section{SCLERIA}

triglomerata, Michx.

verticillata, Muhl.

\section{GRAMINEAE.}

\section{AGROPYRUIM}

caninum, R. \& S. dasystachyum, Vasey.

divergens, Nees.

var. tenue, Vasey.

glaucum, R. \& S., var. occidentale, V. \& S.

repens, $L$.

tenerum, Vasey.

violaceum, Lange.

\section{AGROSTIS}

canina, $\mathrm{L}$.

var. paleata, Vasey, N. var.

exarata, Trin.

geminata, Trin. 
microphylla, Steud. oreophila, Trin. perennans, Tuck. scabra, Willd. varians, Trin. verticillata, Vill. vulgaris, With. var. alba, Vasey.

\section{AIRA}

caryophyllea, L. proecox, $L$.

\section{ALOPECURUS}

alpinus, $\mathrm{L}$.

geniculatus, $\mathrm{L}$.

var. aristulatus, Munro.

var. robustus, Vasey.

Macounii, Vasey. occidentalis, Scrib. pratensis. $L$.

\section{AMMOPHILA}

arundinacea, Host. longifolia, Vasey.

\section{ANDROPOGON}

provincialis, Lam. scoparius, Michx.

\section{ANTHOXANTHUM}

odoratum, $L$.

\section{ARCTAGROSTIS}

latifolia, Griseb.

var. Alaskensis, Vasey.

\section{ARCTOPHILA}

fulva, Rupt.

Læstadii, Rupt. mucronata, Hack.

\section{ARRHENATHERUM}

avenaceum, $P$. B.

\section{ARISTIDA}

basiramea, Engelm. dichotoma, $\mathrm{L}$.

purpurea, Nutt.

\section{ASPRELLA}

hystrix, Willd.

\section{AVENA}

fatua, $L$.

pratensis, var. Americana, Scrib. striata, Michx.

\section{BECKMANNIA}

erucæformis, Host., var. uniflorus, Scrib.

\section{BOUTELOUA}

hirsuta, Lag.

oligostachya, Torr.

racemosa, Lag.

\section{BRACHYELYTRUIM}

aristatum, P. B.

BRIZA

media, $L$.

\section{BROMUS}

Aleutensis, Trin.

breviaristatus, Thurb.

ciliatus, L.

var. ligulatus, Vasey.

var. pauciflorus, Scrib.

Hookerianus, Thurb.

var. minor, Scrib.

Kalmii, Gray.

Macounii, Vasey.

maximus, Desf.

mollis, $L$.

Orcuttianus, Vasey.

Pumpellianus, Scrib.

racemosus, $L$.

secalinus, $L$. 
segetum, Schlecht.

Sitchensis, Bong.

subulatus, Led.

tectorum, $L$.

\section{CATABROSA}

aquatica, P. B.

\section{CENCHRUS}

tribuloides, $\mathrm{L}$.

\section{CHRYSOPOGON}

nutans, Benth.

\section{CINNA}

arundinacea, $\mathrm{L}$.

pendula, Trin.

var. acutiflora, Vasey., N. sp.

var. mutica, Vasey.

\section{CYNODON}

dactylon, Pers.

\section{CYNOSURUS}

cristatus, Linn.

\section{DACTYLIS}

glomerata, $L$.

\section{DANTHONIA}

Californica, Boland.

intermedia, Vasey.

spicata, Beauv.

unispicata, Munro.

\section{DESCHAMPSIA}

alba, Rœm. \& Schultz. atropurpurea, Scheele.

var. latifolia, Scrib.

var. minor, Vasey., N. var. brevifolia, R. Br. cæspitosa, P. B.

var. arctica, Vasey. var. Bottanica, Vasey.

var. longiflora, Trin.

var. maritima, Vasey., N. var. calycina, Presl.

elongata, Munro.

\section{DEYEUXIA}

æquivalis, Benth. \& Hook.

Aleutica, Vasey.

borealis, Macoun., N. sp.

breviaristata, Vasey., N. sp.

Canadensis, Hook.

Columbiana, Macoun., N. sp.

confinis, Kunth.

crassiglumis, Vasey.

deschamsioides, Vasey.

Langsdorffii, Kunth.

Lapponica, Kunth.

Macouniana, Vasey., N. sp.

neglecta, Kunth.

var. Americana, Vasey., N. var.

var. brevifolia, Vasey., N. var

var. robusta, Vasey, N. var.

Pickeringii, Vasey.

Porteri, Vasey.

purpurascens, Kunth.

rubescens, Vasey.

strigosa, Kunth.

Suksdorfii, Scrib.

sylvatica, Kunth.

\section{DISTICHIIS}

maritima, Raf.

var. stricta, Thurb.

\section{DUPONTIA}

Fischeri, R. Br. psilosantha, Rupt.

\section{EATONIA}

obtusata, Gray.

Pennsylvanica, Gray. 


\section{ELYMUS}

Americanus, V. \& S.

arenarius, $\mathrm{L}$.

Canadensis, $\mathrm{L}$. var. glaucifolius, Gray.

Columbiana, Macoun.

condensatus, Presl.

dasystachys, Trin.

Macounii, Vasey.

mollis, Trin.

striatus, Willd.

Vancouverensis, Vasey.

Virginicus, L.

var. submuticus, Hook.

\section{ERAGROSTIS}

major, Host.

minor, Host.

Purshii, Schrad.

reptans, Nees.

\section{FESTUCA}

duriuscula, Lam.

elatior, $L$.

microstachya, Nutt.

var. divergens, Thurb.

var. pauciflora, Scrib.

Myurus, $L$.

nutans, Willd.

occidentalis, Hook.

ovina, L.

var. brevifolia, Watson.

var. polyphylla, Vasey.

var. vivipara, Gray.

Richardsonii, Hook.

rubra, L.

var. villosa, Vasey.

scabrella, Torr.

subulata, Bong.

tenella, Willd.

\section{FLUMINIA}

arundinacea, Fries.
GLYCERIA

angustata, Ledeb.

arctica, Hook.

arundinacea, Kunth.

Canadensis, Trin.

distans, Wahl.

var. airoides, Vasey.

elongata, Trin.

festucæformis, Reich.

fluitans, R. Br.

Lemmoni, Vasey.

maritima, Wahl.

nervata, Trin,

obtusa, Trin.

pallida, Trin.

pauciflora, Presl.

pumila, Vasey.

\section{GRAPHEPHORUIM}

melicoides, Gray.

Wolfii, Vasey.

\section{HIFROCHLOA}

alpina, R. \& S. borealis, R. \& S.

pauciflora, R. Br.

\section{HOLCUS}

lanatus, $L$.

\section{HORDEUIM}

jubatum, L.

maritimum, With.

murinum, L.

pratense, Huds.

pusillum, Nutt.

\section{KGLERIA}

cristata, Pers. var. gracilis, Gray.

var. major, Vasey., N. var.

\section{LEERSIA}

oryzoides, Swartz.

Virginica, Willd. 


\section{LOLIUM}

perenne, $L$.

temulentum, $L$.

\section{MELICA}

acuminata, Boland.

aristata, Thurb.

bulbosa, Geyer.

Harfordii, Boland.

\section{IMILIUIM}

effusum, L.

\section{MUHLENBERGIA}

diffusa, Schreb.

glomerata, Trin.

Mexicana, Trin.

sylvatica, T. \& G.

Willdenovii, Trin.

\section{IMUNROA}

squarrosa, Torr.

\section{ORYZOPSIS}

asperifolia, Michx.

Canadensis, Torr.

cuspidata, Scrib.

melanocarpa, Muhl.

\section{PANICUIM}

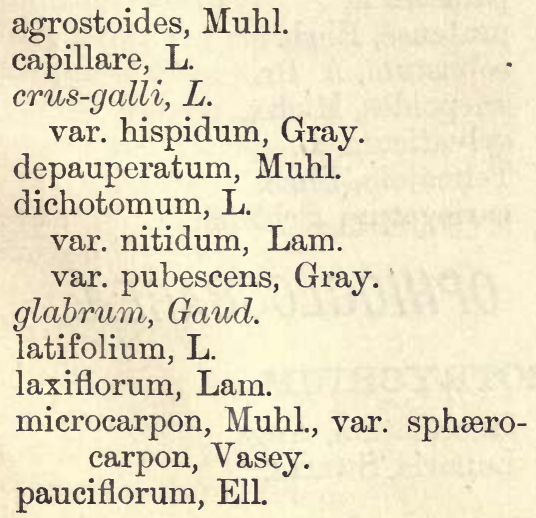

sanguinale, $L$.

scoparium, Lam.

virgatum, $\mathrm{L}$.

xanthophysum, Gray.

\section{PHALARIS}

arundinacea, $\mathrm{L}$.

Canariensis, $L$.

\section{PHIPPSIA}

algida, R. Br.

var. monandra, Kunth.

\section{PHLEUM}

alpinum, $\mathrm{L}$.

pratense, $L$.

\section{PHRAGMITES}

communis, Trin.

\section{PLEUROPOGON}

Sabinii, R. Br.

\section{POA}

abbreviata, R. Br.

alpina, $\mathrm{L}$.

andina, Nutt.

var. purpurea, Vasey., N. var annua, $L$.

Bolanderi, Vasey.

cæsia, Smith.

var. strictior, Gray.

Californica, Vasey.

cenisia, All.

compressa, $L$.

cuspidata, V. \& S.

debilis, Torr.

var. acutiflora, Vasey.

Eatoni, Watson.

flavicans, Ledeb.

glumaris, Trin.

Howellii, V. \& S.

laxa, Hænke.

leptocoma, Trin. 
Macounii, Vasey.

nemoralis, L.

Nevadensis, Vasey.

Nutkaensis, Presl.

pratensis, L.

purpurascens, Vasey.

serotina, Ehrh, stenantha, Trin.

sub-aristata, Scrib.

tenuiflora, Nutt.

var. Oregona, Vasey.

trivialis, $L$.

\section{POLYPOGON}

littoralis, Smith.

Monspeliensis, Desf.

\section{SCHEDONNARDUS}

Texanus, Steud.

\section{SETARIA}

glauca, Beauv.

Italica, Kunth.

verticillata, Beauv.

viridis, Beauv.

\section{SPARTINA}

cynosuroides, Willd.

gracilis, Trin.

juncea, Willd.

polystachya, Willd.

stricta, Roth., var. alterniflora, Gray.

var. glabra, Gray.

\section{SPOROBOLUS}

asperifolius, Thurb. cryptandrus, Gray. cuspidatus, Scrib.

depauperatus, Scrib.

heterolepis, Gray. vaginæflorus, Vasey.

\section{STIPA}

avenacea, Linn.

Columbiana, N. sp. comata, Trin.

Richardsonii, Link. var. major, $\mathrm{N}$. var. spartea, Trin. viridula, Trin.

\section{TRIPLASIS}

purpurea, Chapm.

\section{TRISETUM}

canescens, Buckl. cernuum, Trin. subspicatum, P. B. var. molle, Gray.

\section{ZIZANIA}

aquatica, $\mathrm{L}$.

\section{EQUISETACEAE.}

\section{EQUISETUM}

arvense, $\mathrm{L}$.

hiemale, $\mathrm{L}$.

lævigatum, A. Br.

limosum, L.

littorale, Kuhl.

maximum, Lam.

palustre L.

pratense, Ehrh.

robustum, A. Br.

scirpoides, Michx.

sylvaticum, L.

Telmateia, Ehrh.

variegatum, Schleich.

\section{OPHIOGLOSSACEAE.}

\section{BOTRYCHIUM}

lanceolatum, Angs.

Lunaria, Swartz. 
matricariæfolium, A. Br. simplex, Hitch. ternatum, Swartz. var. dissectum, Milde. var. lunarioides, Milde. var. obliquum, Milde. Virginianum, Swartz.

\section{OPHIOGLOSSUIM}

vulgatum, $\mathrm{L}$.

\section{FILICES.}

\section{ADIANTUM}

pedatum, L.

var. rangiferinum, Burgess.

\section{ASPIDIUM}

acrostichoides, Swartz. aculeatum, Swartz. var. Braunii, Doell. var. scopulinum, Eaton. Boottii, Tuckerm. cristatum, Swartz.

var. Clintonianum, Eaton. Filix-mas, Swartz. fragrans, Swartz. Goldianum, Hook. Lonchites, Swartz. marginale, Swartz. munitum, Kaulf.

Noveboracense, Swartz.

Oreopteris, Swartz. rigidum, Swartz. spinulosum, Swartz var. dilatatum, Hornem. var. intermedium, Eaton. Thelypteris, Swartz.

\section{ASPLENIUIM}

angustifolium, Michx. ebeneum, Ait.

Filix-fœmina, Bernh. var. angustum, Eaton. thelypteroides, Michx.

Trichomanes, $\mathrm{L}$. viride, Huds.

\section{CAMPTOSORUS}

rhizophyllus, Link.

\section{CHEILANTHES}

gracillima, Eaton. lanuginosa, Nutt.

CRYPTOGRAIMME

acrostichoides, R. Br.

\section{CYSTOPTERIS}

bulbifera, Bernh, fragilis, Bernh. montana, Bernh.

\section{DICKSONIA}

pilosiuscula, Willd.

GYMNOGRAMIME

triangularis, Kaulf.

LOMARIA

spicant, Desv. var. serratum, Woll.

\section{ONOCLEA}

sensibilis, L.

var. obtusilobata, Torr. Struthiopteris, Hoffm.

\section{OSIMUNDA}

cinnamomea, L. Claytoniana, $\mathrm{L}$. regalis, $\mathrm{L}$.

\section{PELI画A}

atropurpurea, Link.

densa, Hook.

gracilis, Hook. 
PHEGOPTERIS

alpestris, Mett. calcarea, Fee.

Dryopteris, Fee. hexagonoptera, Fee. polypodioides, Fiee.

\section{POLYPODIUM}

falcatum, Kellogg.

Scouleri, H. \& G.

vulgare, $\mathrm{L}$.

\section{PTERIS}

aquilina, $\mathrm{L}$.

var. lanuginosa, Bong.

\section{SCHIZ阷A}

pusilla, Pursh.

\section{SCOLOPENDRIUM}

vulgare, Smith. var. marginatum, Moore. var. ramosum, Gray.

\section{WOODSIA}

glabella, R. Br.

hyperborea, R. Br.

Ilvensis, $\mathrm{R}$. $\mathrm{Br}$.

obtusa, Torr.

Oregana, Eaton.

- scopulina, Eaton.

WOODWARDIA

Virginica, Smith.

\section{MARSILIACEEE.}

\section{MARSIIIA}

vestita, $H . \&$ G.

\section{SALVINIACEA.}

\section{AZOLIA}

Caroliniana, Willd.

LYCOPODIACEAE.

\section{LYCOPODIUM}

alpinum, L.

annotinum, $\mathrm{L}$.

var. pungens, Desv.

clavatum, $\mathrm{L}$.

complanatum, $\mathrm{L}$.

dendroideum, Michx. inundatum, $\mathrm{L}$.

var. Bigelovii, Tuck. lucidulum, Michx.

sabinæfolium, Willd. Selago, L.

\section{SELAGINELLACEA.}

\section{SELAGINELLA}

apus, Spring.

rupestris, Spring.

selaginoides, Link.

\section{ISOETACEAE.}

\section{ISOETES}

echinospora, Durieu, var.

Braunii, Engelm.

lacustris, $\mathrm{L}$.

maritima, Underwood.

Nuttallii, A. Br.

riparia, Engelm. 


5

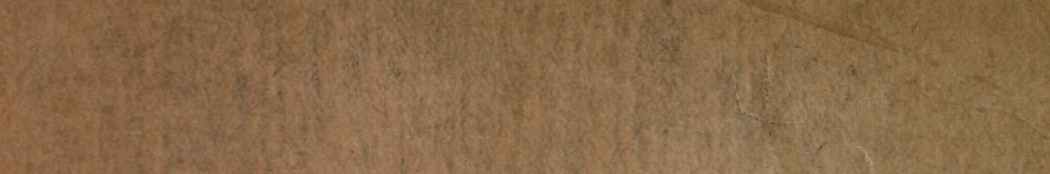

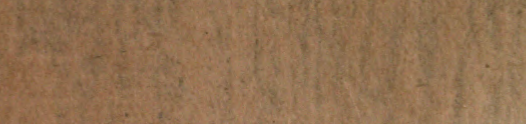

$3.4 x+2 x^{2}$

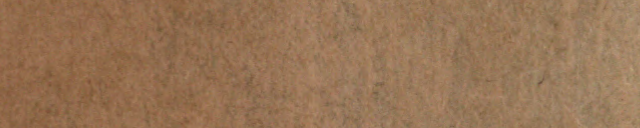

$3(25)=2=$

19.

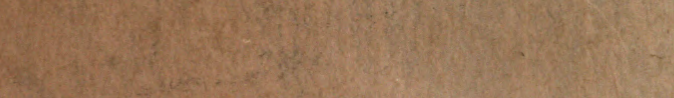

(x)

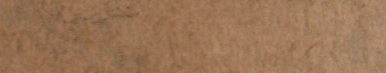
H.

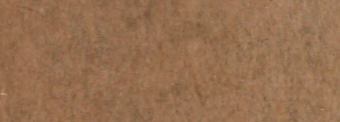

Thes

sit

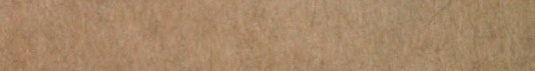

thet

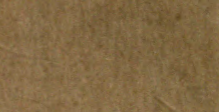

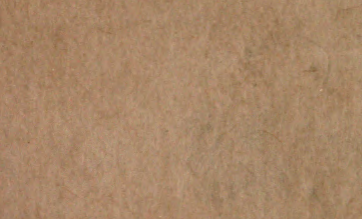

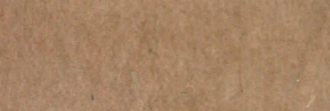
$49+2 x+2 x$

$\cos x$

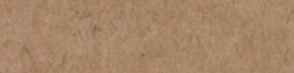
2.

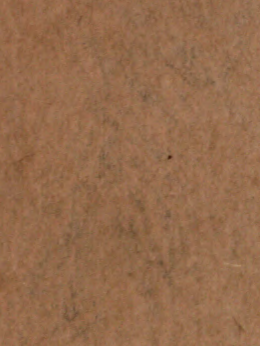

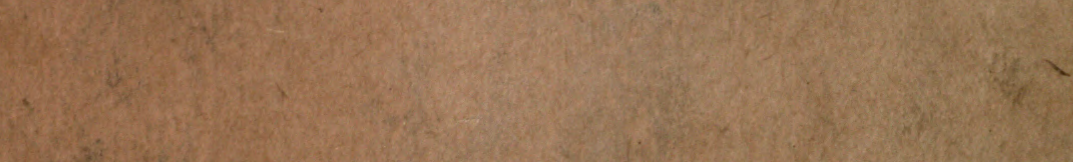

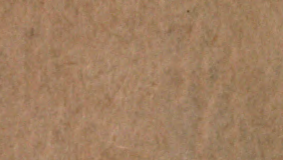

